\title{
II. Der gesetzliche Rahmen der betrieblichen Mitbestimmung 1916 bis 1934
}

\section{Zur Vorgeschichte des Betriebsrätegesetzes}

Das Betriebsrätegesetz vom 4. Februar 1920, das Ende Januar 1920 vom Reichstag nach massiven öffentlichen Protesten und blutigen Zusammenstößen zwischen Demonstranten und Berliner Sicherheitswehr mit den Stimmen der Weimarer Koalition angenommen wurde, war zugleich ein Kind der Revolution wie der deutschen Tradition zur Verrechtlichung der industriellen Beziehungen im Betrieb, die sich bis zu den Verhandlungen der Frankfurter Nationalversammlung von 1848 zurückverfolgen läßt. ${ }^{1}$ Gewerbeordnungs- und Bergrechtsnovellen brachten noch vor dem 1. Weltkrieg die Einrichtung fakultativer Arbeiterausschüsse in der Industrie sowie die obligatorische Einrichtung von Arbeiterausschüssen im preußischen Bergbau. ${ }^{2}$ Die Erfahrungen mit diesen Ausschüssen waren unterschiedlich, vor allem auf Arbeiterseite wurden deren geringe Autonomie, der mangelnde Entlassungsschutz, die häufig undemokratischen Wahlverfahren und die geringe Konfliktbereitschaft der zumeist in den Ausschüssen federführenden "gelben Werkvereine " kritisiert. ${ }^{3}$ Ähnlich argumentierten SPD und freie Gewerkschaften, die auch generelle Vorbehalte gegenüber Harmoniekonzepten im Betrieb hegten und ein Primat von Partei und Gewerkschaft im Klassenkampf vertraten. ${ }^{4}$ Die öffentliche Meinung im Kaiserreich war unschlüssig; zwar breiteten sich die fakultativen Arbeiterausschüsse mehr und mehr aus und allgemein billigte man ihnen eine positive Funktion bei der Erhaltung des sozialen Friedens im Betrieb zu. Angesichts des geringen Entgegenkommens der Arbeitgeber führten aber die „Ausschüsse ein Scheindasein und (verfielen) einer ruhmlosen Vergessenheit", wie der Berichterstatter des Handwörterbuchs der Staatswissenschaften 1909 konstatierte. ${ }^{5}$ Besserung erwartete man nicht durch neue Gesetze: „Es wäre offenbar Widersinn, dem Fabrikanten einen Arbeiterrat aufzudringen. Selbst wenn man es könnte, würde man ihn keinesfalls dazu zwingen können, den ihm erteilten Rat wirklich zu befolgen, also käme man über eine platonische Beratung gewisser Fra-

1 Vgl. Muszynski, Wirtschaftliche Mitbestimmung, S. $28 \mathrm{ff}$. Teuteberg, Geschichte der industriellen Mitbestimmung in Deutschland. Feig, Betriebsräte, in: HdStW, 4. Aufl., Bd. 2, S. 625-633.

2 Costas, Anfänge der Partizipation, in: Bergmann u. a. (Hg.), Geschichte als politische Wissenschaft, S. 335-378. Kroker, Arbeiterausschüsse, in: Anschnitt 30 (1978), S. 204-215. Rupieper, Kooperation und Konflikt, in: IWK 20 (1984), S. 347-373. Kocka, Unternehmensverwaltung, S. 347363. Stieda, Arbeitsordnungen und Arbeiterausschüsse, in: HdStW, 3. Aufl., Bd. 1, S. 1140-1149.

3 Brüggemeier, Leben vor Ort, S. 217-220. Kocka, Unternehmensverwaltung, S. 357-363. Ein interessantes Schlaglicht bei Duisberg, Die Arbeiterschaft der chemischen Großindustrie, S. $42 \mathrm{f}$.

+ Costas, Anfänge der Partizipation, S. 344 f.

5 Stieda, Arbeitsordnungen und Arbeiterausschüsse, S. 1148. 
gen nicht heraus. Als freiwillige Einrichtung werden die Ausschüsse erst dann eine gedeihliche Wirksamkeit entfalten können, wenn in den Anschauungen der Fabrikanten ein völliger Umschwung sich vollzogen haben wird." ${ }^{\text {6 }}$

Die „Fabrikanten“" waren aber zu diesem Entgegenkommen noch im Krieg, als die Kooperation der Gewerkschaften selbst Staat und Militär zu sozialpolitischen Kompromissen veranlaßte, nicht bereit. Das Vaterländische Hilfsdienstgesetz vom Dezember 1916, von Gerald D. Feldman als Triumph der Arbeiterschaft bezeichnet, schrieb daher gegen den erklärten Willen vor allem der rheinisch-westfälischen Großindustrie die obligatorische Einrichtung von Arbeiterausschüssen in sog. Hilfsdienstbetrieben, d.h. freizügigkeitsbeschränkten rüstungswichtigen Betrieben mit mehr als 50 Beschäftigten, fest und erlaubte erstmals die Bildung von unabhängigen betrieblichen Interessenvertretungen der Arbeiterschaft.? Diese Arbeiterausschüsse hatten nach dem Wortlaut des Gesetzes das "gute Einvernehmen innerhalb der Arbeiterschaft des Betriebs zu fördern“ und „Anträge, Wünsche und Beschwerden der Arbeiterschaft, die sich auf die Betriebseinrichtungen, die Lohn- und sonstigen Arbeitsverhältnisse des Betriebs und seiner Wohlfahrtseinrichtungen beziehen, zur Kenntnis des Unternehmers zu bringen und sich darüber zu äußern. ${ }^{8}$ Diese Regelung stellte noch keine entscheidende Verbesserung der Rechte und Interventionsmöglichkeiten der alten, fakultativen Arbeiterausschüsse dar. Das Gesetz beinhaltete aber zwei entscheidende Neuerungen. Zunächst konnten nach den Wahlordnungen der jeweiligen Bundesstaaten die Gewerkschaften sich in den Betrieben mit eigenen Listen zur Wahl stellen und damit in der Tat vom Arbeitgeber unabhängige Ausschüsse sichern. Zum anderen bestand die Möglichkeit, bei ungelösten betrieblichen Konflikten, wobei explizit auch die Lohnfrage einbezogen war, eine aus Arbeitgeber- und Gewerkschaftsvertretern paritätisch besetzte Schiedsstelle anzurufen, die das Hilfsdienstgesetz für die Regelung der Arbeitsmarkt- und Beschäftigungsverhältnisse der Hilfsdienstpflichtigen eingeführt hatte. ${ }^{9}$

Trotz erheblicher Anfangsschwierigkeiten wurden die Vorschriften des Hilfsdienstgesetzes im Laufe der Jahre 1917 und 1918 durchgesetzt: die Gewerkschaften erhielten Zugang zu den Großbetrieben; die Aushandlung der Lohn- und Arbeitsbedingungen wurde zumindest in den Betrieben nach und nach „kollektiviert". Die Funktionsprobleme der Arbeitsmärkte aber wurden hierdurch nicht geringer: die Zahl der betrieblichen Lohnkonflikte nahm vielmehr stark zu, die Arbeiterausschußverhandlungen konnten kollektive Lohnregelungen auf Branchenebene nicht ersetzen, sondern schienen sie im Gegenteil nur um so dringli-

6 Ebenda, S. 1149.

Gesetz über den Vaterländischen Hilfsdienst vom 5. 12. 1916, in: RGBI. 1916, Nr. 276, S. 1333, $1335 \mathrm{ff}$. Zum Hilfsdienstgesetz vgl. Feldman, Armee, Industrie und Arbeiterschaft, S. $169 \mathrm{ff}$. Zur Haltung der rheinisch-westfälischen Industrie, insbesondere Großchemie und Schwerindustrie sowie zu ihrem diesbezüglichen Konflikt mit den Militärbehörden siche Niederschrift über die Besprechung im Industrie-Club zu Düsseldorf, 19. 8. 1917, BAL 216/4. Neben den unterschiedlichen Auffassungen von Kirdorf und Duisberg einerseits, den Militärbehörden andererseits wurden auf dieser Tage auch innerindustrielle Konflikte deutlich, da sich Paul Silverberg im Gegensatz zu Großchemie und Schwerindustrie nachhaltig für eine Kooperation mit den Gewerkschaften einsetzte.

8 Gesetz über den Vaterländischen Hilfsdienst $\$ 12$, RGBI. 1916, Nr. 267, S. 1335.

9 Hilfsdienstgesetz $\$ 8,9$. 
cher zu machen. Die Kritik der Arbeitgeber an der Praxis der im übrigen gegen Repression kaum geschützten Arbeiter- und der Schlichtungsausschüsse schwoll folgerichtig rasch an, während sich insbesondere Vetreter der oberen Reichsbehörden zufrieden über das Gesetz äußerten. Eine endgültige Lösung der betrieblichen Arbeitsmarktprobleme konnte das Gesetz indes allein deshalb nicht sein, da es sich um ein Kriegsnotgesetz handelte. Im Reichswirtschaftsamt machte man sich daher 1918 daran, die vorhandenen Überlegungen zur zukünftigen Betriebsverfassung zusammenzufassen und in einem Gesetzentwurf zu fixieren. Zunächst plädierte man für eine dauerhafte Verankerung der Arbeitsausschüsse und ihrer Rechte in der Gewerbeordnung. Widerstände von Seiten der Unternehmen gegen ein derartiges Vorgehen erwartete man in völliger Verkennung der Realität nicht. Auch wenn man wenig später ein eigenes Gesetz favorisierte, hielt man an der Vorstellung, die Vorschriften des Hilfsdienstgesetzes hätten sich bewährt, fest. ${ }^{10}$

Die Arbeiten des Reichswirtschaftsamtes wurden von der Revolution überholt. Wenige Tage nach der Revolution, am 12. November 1918, wurde das Hilfsdienstgesetz aufgehoben. Am 15. November 1918 brachte das Zentralarbeitsgemeinschaftsabkommen eine neue Geschäftsgrundlage für die Arbeiterausschüsse: „Für jeden Betrieb mit einer Arbeiterschaft von mindestens 50 Beschäftigten ist ein Arbeiterausschuß einzusetzen, der diese zu vertreten und in Gemeinschaft mit dem Betriebsunternehmer darüber zu wachen hat, daß die Verhältnisse des Betriebes nach Maßgabe der Kollektivvereinbarungen geregelt werden. "11 Diese Vereinbarung war alles andere als konkret. Sie führte zwar häufig zur Absetzung der bisherigen Arbeiterausschüsse, die nach der Wahlordnung des Hilfsdienstgesetzes ins Amt gekommen waren und nun vielen Belegschaften und Teilen der Gewerkschaftsbewegung nicht mehr entschieden genug auftraten. ${ }^{12}$ Die neu ins Amt kommenden Interessenvertreter hatten aber keinerlei Richtschnur, an der sie ihr Verhalten und Handeln ausrichten konnten - die Praxis der Betriebsvertretungen wurde zunehmend unübersichtlich, zumal auch die Reaktion der Werksleitungen auf die neuen, im strengen Sinne nicht legalen betrieblichen Interessenvertretungen unterschiedlich ausfiel. Hinzukam, daß sich ein Großteil der neuen Interessenvertretungen explizit politisch verstand und in eine mehr oder weniger offene Organisationskonkurrenz zu den Gewerkschaften trat bzw. ihnen wichtige Kompetenzen bestritt. ${ }^{13}$

Die Frage der zukünftigen Organisation der betrieblichen Interessenvertretungen konnte solange nicht entschieden werden, wie nicht innerhalb der Arbeiterbewegung selbst geklärt war, welche Bedeutung die betrieblichen Arbeitervertretungen in Zukunft haben sollten. ${ }^{14}$ Erst die Entscheidung für die parlamentarische Republik und damit explizit gegen eine politische Räteorganisation durch eine Mehrheit von MSPD und Gewerkschaften auf dem ersten Kongreß der Arbeiter-

10 Entwurf zur Änderung der Gewerbeordnung vom 27. 7. 1918; Entwurf eines Gesetzes betr. obligatorische Arbeiterausschüsse 4. 9. 1918, GStA Merseburg Rep 120 BB VII 1 Nr. 9 adh. 3, Bd. 1, Bll. 171-176, 178-189.

11 Zentralarbeitsgemeinschaftsabkommen vom 15.11. 1918, abgedruckt bei Feldman, Steinisch, Industrie und Gewerkschaften, S. 135.

12 Costas, Anfänge der Partizipation, S. 362 f.

13 Ebenda.

14 Hierzu ausführlich Oertzen, Betriebsräte. 
und Soldatenräte in Berlin vom 16.-20. Dezember 1918 brachte die nötige Klarheit, die der Staatssekretär des Reichsarbeitsamtes Bauer mit der Verordnung vom 23. Dezember 1918 über Tarifverträge, Arbeiter- und Angestelltenausschüsse und Schlichtung von Arbeitsstreitigkeiten postwendend umsetzte. ${ }^{15}$ Die Verordnung verband die rechtliche Tradition, deren letzter Ausdruck der Gesetzentwurf des Reichswirtschaftsamtes vom September 1918 war, mit der gewerkschaftlichen Vorstellung, die Betriebsvertretungen seien als gewerkschaftlicher Vorposten im Betrieb vor allem eine Kontrollinstanz zur Einhaltung der Tarifabkommen. Sie kehrte die Aufgabenstellung der Arbeiterausschüsse gegenüber dem letzten Gesetzentwurf des Reichswirtschaftsamtes, aus dessen einschlägigen Abteilungen im übrigen das Reichsarbeitsamt seinen bürokratischen Kern bezogen hatte ${ }^{16}$, um und setzte die Interessenvertretung und Überwachung der Tarifvereinbarungen an die Spitze der Aufgaben der Betriebsvertretungen. Sollten tarifliche Regelungen nicht existieren, gestand die Verordnung den Ausschüssen sogar das Recht zu eigener Verhandlungsführung mit den Unternehmen, freilich in Absprache mit den Gewerkschaften zu. Die Förderung des sozialen Friedens, der Unfall- und Arbeitsschutz sowie die Unterstützung der Behörden folgten als Aufgaben erst an zweiter Stelle. ${ }^{17}$ Die Zahl der betroffenen Betriebe wurde zudem erweitert; nach der Verordnung waren nunmehr alle Betriebe mit mehr als 20 Beschäftigten ausschußpflichtig. Das Schlichtungsverfahren wurde im Prinzip beibehalten. Neben die bisherige öffentliche, paritätisch zusammengesetzte Schlichtung traten allerdings nun tarifliche, freiwillige Schlichtungsinstanzen für Konflikte, die sich mit oder über Tarifregelungen ergaben. ${ }^{18}$ Ebenso wie dem Hilfsdienstgesetz fehlte der Dezemberverordnung eine genauere Benennung der Aufgaben, Rechte und Pflichten der Ausschüsse. Auch war kein Passus in der Verordnung enthalten, der die betrieblichen Interessenvertreter vor unternehmerischer Willkür schützte. Angesichts einer derartigen Konstellation war es kein Wunder, daß die Praxis der im Laufe des Jahres 1919 gewählten Ausschüsse durch die Verordnung nur gering beeinflußt wurde, vor allem aber, daß sich radikale Belegschaften und Gewerkschaftsorganisationen hierdurch nicht gebunden fühlten. ${ }^{19}$

Vor diesem Hintergrund gewann die Rätefrage im Laufe des Frühjahres 1919 ein eigenständiges, auch theoretisch-konzeptionell großes Gewicht. ${ }^{20}$ Bei den großen Arbeitskämpfen an der Ruhr und in Mitteldeutschland im Frühjahr 1919 bildeten die Räte als scheinbar angemessene Organisationsform basisnaher Radikalität ein alternatives Organisationsmodell nicht nur zur Führung von Arbeitskämpfen mit „syndikalistischem Hintergrund“, sondern für Staat und Wirtschaft schlechthin. Diesem Druck der Streikbewegungen glaubte die Reichsregierung nur durch Zugeständnisse entgegenkommen zu können, die den Rätegedanken aufgriffen. Nachdem die Reichsregierung im Februar 1919 in Verhandlungen mit den Vertretern der streikenden Bergarbeiterschaft den Betriebsratsgedanken für

${ }_{15}$ Winkler, Von der Revolution zur Stabilisierung, S. 97-113.

16 Zur Geschichte des Reichsarbeitsministeriums siehe Deutsche Sozialpolitik 1918-1928, S. $5 \mathrm{ff}$.

17 Verordnung vom 23. 12. 1918, RGBl. 1918, Nr. 287, S. $1456 \mathrm{f}$.

18 Ebenda, $\$ 20$.

19 Costas, Anfänge der Partizipation, S. 363. Oertzen, Betriebsräte, S. 127. Winkler, Von der Revolution zur Stabilisierung, S. $114 \mathrm{ff}$.

20 Oertzen, Betriebsräte, laufend. 
den Bergbau akzeptiert hatte, wurde bereits im Mai 1919 der Entwurf eines Betriebsrätegesetzes im Reichsarbeitsministerium vorgelegt und mit den Gewerkschaften und wirtschaftlichen Interessenverbänden diskutiert. ${ }^{21}$ Von Seiten der Arbeitgeberverbände, aber auch und insbesondere von Seiten der freien Gewerkschaften wurden dabei frühzeitig Widerstände gegen bestimmte, im Entwurf vorgesehene Rechte der Betriebsvertretungen angekündigt: Störten die Arbeitgeber die zu weite Fassung der Rechte der Betriebsräte, die „niedrigen Schranken“ beim passiven Wahlrecht, die Einblicksmöglichkeiten in Betriebsgeheimnisse und vor allem die nicht unbedeutenden Rechte der Belegschaften selbst, so hatten die freien Gewerkschaften Probleme mit der zu engen Bindung der Betriebsvertretungen an die Belegschaften und ihre relative Autonomie von den Gewerkschaften. Im Gegensatz zu den Arbeitgebern sahen die freien Gewerkschaften den Entwurf aber "grundsätzlich als ausreichend" an, da der Primat der Gewerkschaften vor den Betriebsvertretungen vom Text her gesichert war. ${ }^{22}$ Das Fehlen wirtschaftlicher Mitbestimmungsrechte im ersten Entwurf traf indes auf erhebliche Proteste zahlreicher betrieblicher Interessenvertretungen und insbesondere der freien Angestelltengewerkschaften, auf deren Druck hin in den zweiten Entwurf des Gesetzes das Recht der Betriebsvertretungen aufgenommen wurde, ein bis zwei Vertreter in die Aufsichtsorgane großer Kapitalgesellschaften zu entsenden. ${ }^{23}$ Die zweite Fassung wurde daher von den Arbeitgebern mit der Begründung abgelehnt, daß die Mitbestimmungsrechte des Betriebsrates eine „ernste $\mathrm{Ge}$ fahr" für die "unerläßliche Gemeinschaftsarbeit, weil auf völliger Verkennung ihrer natürlichen Grundlagen beruhend", darstellten. Die "Gemeinschaftsarbeit" vertrage sich nicht mit Eingriffen in die Geschäftsführung; die zu weitgehende Einsicht in die Geschäftsunterlagen komme einer Preisgabe von Geschäftsgeheimnissen gleich. Man unterzog den Entwurf einer genauen Analyse und legte de facto einen eigenen Gesetzentwurf vor, dessen Realisierung die Betriebsräte zu hilflosen Anhängseln der Unternehmensleitungen gemacht hätte. ${ }^{24}$

Trotz der allseitigen Widerstände - im Juni 1919 begann eine reichsweite Kampagne von Interessenverbänden und Industrie- und Handelskammern gegen den Gesetzentwurf ${ }^{25}$ - und der nur lauen Unterstützung der freien Gewerkschaften hielt das Kabinett Bauer an seiner im Frühjahr verkündeten Absicht fest, das Gesetz schnell zu verabschieden. ${ }^{26}$ Ziel der Regierung blieb eine Kanalisierung der Rätebewegung, und dazu schien der vorliegende Gesetzentwurf das geeignete In-

21 Niederschrift über eine Besprechung im Reichsarbeitsministerium, 15. 5. 1919, GStA Merseburg Rep 120 BB VII 1 Nr. 9 adh. 5, Bd. 1, Bll. 4-13.

22 Siehe auch dic Zusammenfassung der ersten Gesetzgebungsphase in: Akten der Reichskanzlei, Kabinett Bauer, S. 129, Anm. 1.

23 Siehe hierzu Müller, Strukturwandel und Arbeitnehmerrechte, S. 120-122.

24 RDI, VDA etc. an Mitglieder der Nationalversammlung, Juli 1919, betr.: Entwurf eines Gesetzes über Betriebsräte, GStA Merseburg Rep 120 BB 1 Nr. 9 adh. 3, Bd. 1, Bll. 449-469.

25 Vgl. die Eingabensammlung in GStA Rep 120 VII 1 Nr. 9 adh. 5, Bd. 1, laufend. Siehe ferner den Verweis auf die Eingaben an die Reichskanzlei, in: Akten der Reichskanzlei, Kabinett Bauer, S. $129 \mathrm{f}$.

26 Siehe Arbeitsprogramm des Kabinetts, Kabinettsitzung 3. 7. 1919: „Das System der Arbeiter- und Betriebsräte soll mit Beschleunigung durchgeführt werden; das Gesetz über die Betriebsräte soll noch im Juli eingebracht und möglichst noch vor den Ferien verabschiedet werden." Akten der Reichskanzlei, Kabinett Bauer, S. 49. 
strument, obwohl wegen der widersprüchlichen Interessen eine rasche Beratung des Gesetzes immer unwahrscheinlicher wurde: „Von vielen Seiten“, so wurde am 16. Juli 1919 in der Reichskanzlei notiert, „wurde Protest dagegen erhoben, daß der Entwurf überhastet durchberaten werden müsse und den Interessenten nicht genügend Zeit zur Bearbeitung des Entwurfs gelassen werde. ${ }^{27}$ Am 30. Juli 1919 beriet das Reichskabinett den Gesetzentwurf des Reichsarbeitsministeriums, der in seinen Grundzügen vom Reichsarbeitsminister erläutert wurde. Hiernach sollten in Betrieben mit mehr als 20 Beschäftigten Betriebsräte gebildet werden, in denen entsprechend der Belegschaftsstruktur Arbeiter und Angestellte proportional vertreten sein müßten. In sozial- und personalpolitischer Hinsicht hatte der Betriebsrat echte Mitbestimmungsrechte, in wirtschaftlicher hingegen nicht, sieht man von der gleichberechtigten Vertretung von Betriebsratsangehörigen in den jeweiligen Aufsichtsräten ab, worin das Reichsarbeitsministerium freilich einen Durchbruch auf dem Weg von der Mitwirkung zur Mitbestimmung erblickte. ${ }^{28}$ Das Kabinett diskutierte den Entwurf nicht kontrovers. Von der Preußischen Regierung kamen hingegen Einwände ${ }^{29}$, auch verschiedene Reichsministerien betonten das Problem der Anwendung des BRG im öffentlichen Dienst. ${ }^{30}$ Man einigte sich darauf, zu diesen Problemen Referentenbesprechungen durchzuführen, die noch Ende Juli/Anfang August 1919 stattfanden. Am 5. August 1919 stand der Gesetzentwurf erneut auf der Tagesordnung des Kabinetts, die Beratung wurde aber vertagt, nachdem der Preußische Minister für Handel und Gewerbe festgestellt hatte, „der Entwurf bedeute den organisierten Bolschewismus“, da er nur die Rechte, nicht auch die Pflichten der Betriebsvertretungen festschreibe. ${ }^{31}$ Dieser Vorwurf war absurd, doch markierte er den behördeninternen Diskussionsstand. Die Berichte der Gewerbeaufsichtsbehörden, die im Sommer 1919 noch mit der Durchsetzung der Dezemberverordnung von 1918 beschäftigt waren, quollen von Schilderungen chaotischer Verhältnisse in den Betrieben über. ${ }^{32}$ Insofern war es nicht verwunderlich, wenn die Preußische Regierung, bei der sich die industriellen Protesteingaben in ihrer Wirkung mit den Berichten der Gewerbeauf-

27 Vermerk des Geheimen Regierungsrats Schlettwein über den Entwurf eines Betriebsrätegesetzes, 16. 7. 1919, Akten der Reichskanzlei, Kabinett Bauer, S. 129 f.

28 Drucksache Nr. 928 der verfassunggebenden Deutschen Nationalversammlung, S. 22, hier zit. nach: BAP RAM 489, Bl. 242. Siehe zu den Gesetzentwürfen GStA Merseburg Rep 120 BB VII 1 Nr. 9 adh 5 gen., Bd. 1, Bll. 299 ff. Zur Kabinettsitzung Akten der Reichskanzlei, Kabinett Bauer, S. $156-158$.

29) Siehe Votum des Preuß. Ministers des Innern zum Entwurf des BRG, 11. 8. 1919, GStA Merseburg Rep 120 BB VII 1 Nr. 9 adh. 5 gen., Bd. 1, Bll. 117-120. Der PrMdI kritisierte namentlich die jährliche Wiederwahl, das zu niedrige Wahlalter und damit die Begünstigung junger und unreifer Elemente, das Recht der Betriebsversammlung zur Abberufung der Betriebsräte und riet zu einer Vertagung des ganzen Gesetzgebungsprozesses. Andernfalls werde Preußen einen eigenen Gesetzentwurf vorlegen.

30 Siehe hierzu ausführlich BAP RAM 468f. Es gehört zu den interessanten, im Kontext dieser Arbeit nicht weiterverfolgten Gesichtspunkten des Betriebsrätegesetzes, daß u. a. die Reichs- und Landesministerien, die in vielen Fällen die Aufsicht über die Einhaltung des BRG in der Privatwirtschaft zu führen hatten, ihrerseits die Rechte der Personalvertretungen so eng wie möglich halten wollten. Große Energien mußte das RAM daher vor allem auf die Erarbeitung und gemeinsame Verabschiedung von Durchführungsverordnungen zum BRG im Behördenbereich verwenden.

31 Kabinettssitzung vom 5. 8. 1919, Akten der Reichskanzlei, Kabinett Bauer, S. $168 \mathrm{f}$.

32 Siehe die Sammlung der Berichte betreffend Arbeiter- und Angestelltenausschüsse, GStA Merseburg Rep 120 BB VII 1 Nr. 9 adh. 3 Slg II, laufend. 
sichtsbeamten ergänzten, der ganzen vom Reich ins Auge gefaßten revolutionskompensatorischen Betriebsrätegesetzgebung mit Skepsis gegenüberstand. Die Reichsregierung blieb indes bei ihrer Linie; das Kabinett verabschiedete den Entwurf am 7. August $1919 .{ }^{33}$ Kurz danach wurde er im Ältestenausschuß der Nationalversammlung behandelt: die erste Lesung in der Nationalversammlung wurde für die Zeit nach dem 20. August 1919 ins Auge gefaßt. Im Reichsrat fanden Beratung und Verabschiedung des Entwurfs am 16.August 1919 statt. Einer zügigen Behandlung in der Nationalversammlung schien nichts mehr im Wege zu stehen. ${ }^{34}$

Das Gegenteil trat ein. Die Verhandlungen um den Versailler Friedensvertrag verzögerten den Gesetzgebungsprozeß. Die in sich zerstrittene DDP, die wegen des Versailler Vertrages aus der Regierungskoalition ausgeschieden war, später im September 1919 - aber über ihren Wiedereintritt verhandelte und danach wieder an der Regierungskoalition teilnahm, nutzte ihre Schlüsselstellung aus, um in den Verhandlungen des sozialpolitischen Ausschusses der Nationalversammlung die Einwände der Industrie aufzugreifen und in langwierigen Debatten Veränderungen des Betriebsrätegesetzes durchzusetzen, die mit dem Argument, das Gesetz behandle die deutsche Wirtschaft zu schematisch, auf eine Beseitigung des Einflusses der Betriebsvertretungen auf die wirtschaftliche Führung der Unternehmen hinausliefen. ${ }^{35}$ Obwohl der wirtschaftliche Einfluß der Betriebsvertretungen im Gesetzentwurf nur gering war, erwiesen sich die Interventionen der organisierten Industrie, der „bürgerlichen" Regierungsparteien und verschiedener Reichs- und Preußischer Behörden als erfolgreich: sie beseitigten jene Reste im Gesetzentwurf, die in der einen oder anderen Form auf den Druck der streikenden und nach grundlegenden Veränderungen drängenden Arbeiterschaft zurückzuführen waren. ${ }^{36}$ Lediglich die Aufsichtsratsvertretung der Betriebsräte blieb gegen den Widerstand der Arbeitgeber und von Teilen der DDP - im Gesetzestext erhalten, sollte jedoch durch ein gesondertes Gesetz präzisiert werden. Die Gründe für das Festhalten an der Aufsichtsratsmitbestimmung waren dabei nicht allein taktischer Natur, sondern gaben dem sozialpolitischen Gestaltungswillen nicht zuletzt der christlich-sozialen Strömungen Ausdruck. Die Begründung des Gesetzentwurfes bezeichnete die Aufsichtsratsmitbestimmung nachdrücklich als eine „Neuerung völlig grundlegender Art“, als Verwandlung des im „allgemeinen gewährten Mitberatungsrechts in ein Mitbestimmungsrecht", das vorgeschlagen werde „in der Überzeugung, daß nichts so sehr die Arbeitsfreudigkeit, das Verantwortlichkeitsgefühl und das Interesse an der Hebung der Betriebsleistungen und des Ertrages zu steigern geeignet ist, als die verantwortliche Mitwirkung an der obersten Leitung des Unternehmens, daß solche jeder äußerlichen Kontrolle bei weitem überlegen ... ist. ${ }^{\text {}}{ }^{37}$ Zumindest für die federführenden Beamten des

33 Akten der Reichskanzlei, Kabinett Bauer, S. 170.

34 Kabinettssitzung vom 14. 8. 1919: 5. Mitteilungen über die Besprechung im Ältestenausschuß mit handschriftlichem Nachtrag über die Behandlung im Reichsrat, Akten der Reichskanzlei, Kabinett Bauer, S. 276.

35 Vgl. hierzu Mommsen, Verspiclte Freiheit, S. 77-79; Oertzen, Betriebsräte; Gloria Müller, Strukturwandel und Arbeitnehmerrechte, S. 116-120; Winkler, Von der Revolution zur Stabilisierung, S. 283-288.

36 Mommsen, Verspielte Freiheit, S. 86-94.

37 Überarbeiteter Gesetzentwurf mit Begründung, BAP RAM 489, Bll. 240-245. 
Reichsarbeitsministerium begründete nicht zuletzt die Aufsichtsratsmitbestimmung eine neue Qualität der Industriellen Beziehungen und war jenseits aller Taktik deutlichster Ausdruck der neuen Zeit, wie es vor allem Reichsarbeitsminister Brauns $^{38}$ in den späteren Verhandlungen um das Aufsichtsratsgesetz nicht müde wurde zu betonen. Das im Januar 1920 endgültig angenommene Betriebsrätegesetz $^{39}$ war trotz der Abstriche, die sukzessive am Gesetzeswerk hatten gemacht werden müssen, ein Kind der Revolution, da es ohne sie weder seinen Namen erhalten hätte noch in derart kurzer Zeit verabschiedet worden wäre. Auch der Ansatz zu wirtschaftlicher Mitbestimmung über die Aufsichtsratsvertretung wäre ohne die revolutionären Bedingungen und den Druck der „Verhältnisse“ kaum in den Gesetzestext aufgenommen worden. In seiner Substanz griff es allerdings auf die Tradition der Arbeiterausschüsse zurück, deren Rolle und Funktion seit 1916 sukzessive ausgeweitet worden war und Mitte 1918 Niederschlag in ersten entsprechenden Gesetzentwürfen gefunden hatte. Sein zentraler Gedanke, die Verbindung von Effizienzsteigerung und Interessenvertretung, verband sich entschieden mit der Tradition bürgerlicher Sozialreform, die im Reichsarbeitsministerium und seinen Vorgängerbehörden das konzeptionelle Denken bestimmte.

Diese Verbindung von sozialreformerischer Tradition und revolutionärem Kontext bedingte die politische Interpretation des Gesetzes. Für einen Großteil der radikalen Arbeiterschaft, die USPD und zahlreiche Gewerkschaftsorganisationen war es schlichter Betrug: „In diesem Betriebsrätegesetz ist der Gedanke des Rätesystems, wie ihn die klassenbewußte Arbeiterschaft vertritt, ins Lächerliche verzerrt worden“, erklärte am 14. Januar 1920 der USPD-Abgeordnete Geyer in der Nationalversammlung und fuhr fort: „Der Name ,Betriebsrätegesetz' soll nur dazu dienen, um Verwirrung in den Köpfen der Arbeiterschaft anzurichten. ${ }^{40}$ Für Geyer wie für viele andere linke Gewerkschafter war das Betriebsrätegesetz ein Kniefall von Regierung und Parlament vor dem Kapital. Dessen Vertreter sahen das freilich völlig anders und erblickten selbst in dem entschärften Gesetz einen Angriff auf die Grundlagen des freien Unternehmertums, zumal die Vertretung der Betriebsräte im Aufsichtsrat trotz aller Interventionen nicht hatte verhindert werden können. ${ }^{41}$ Seine wärmsten Befürworter hatte das Gesetz bei den christlichen Gewerkschaften; die Mehrheit der freien Gewerkschaften hatte sich mit dem Gesetz abgefunden, da es ihren Organisations- und Kompetenzbereich nicht tangierte, im Gegenteil die Rolle der Gewerkschaften gegenüber den betrieblichen Interessenvertretungen erheblich aufwertete (Beratungsrecht, Benehmenszwang in Tarif- und Lohnfragen etc.). ${ }^{42}$

Das Betriebsrätegesetz blieb neben der Einrichtung des Vorläufigen Reichswirtschaftsrates und der Einführung "gemeinwirtschaftlicher" Strukturen im

38 Zu Brauns Mockenhaupt, Weg und Wirken. Ferner Deuerlein, Heinrich Brauns, in: Hermens, Schieder (Hg.), Staat, Wirtschaft und Politik in der Weimarer Republik, S. 41-96.

39 Reichsgesetzblatt 1920, Nr. 26, S. $147 \mathrm{ff}$.

40 Verhandlungen der Verfassungsgebenden Deutschen Nationalversammlung, Sten. Berichte, 136. Sitzung, 14. 1. 1920, S. $4249 \mathrm{f}$.

41 Große Protestkundgebung des Reichsverbandes der Deutschen Industrie, 11.12. 1919. Vgl. auch Feldman, Steinisch, Industrie und Gewerkschaften, S. 54.

42 Brauer, Das Betriebsrätegesetz und die Gewerkschaften. 
Bergbau $^{43}$, die freilich ebensowenig zur Mitbestimmung der Gewerkschaften in wirtschaftlichen Unternehmensentscheidungen führten wie der Reichswirtschaftsrat zur Wirtschaftsdemokratie, die einzige gesetzliche Konkretisierung des Paragraphen 165 der Reichsverfassung, der unter dem Eindruck der revolutionären Ereignisse die Gleichberechtigung der Arbeiterschaft in Wirtschaft und Gesellschaft postuliert hatte. Die Bestimmungen des Betriebsrätegesetzes waren indes in einer Weise konzipiert, so die spätere Auffassung vieler Freigewerkschafter und ihnen nahestehender Arbeitsrechtler, daß sie nur im Rahmen gemeinwirtschaftlicher Strukturen Sinn machten. Unterstützung des Betriebswohls und Aufsichtsratsmitbestimmung verwiesen in dieser Sicht zwingend auf ihre gemeinwirtschaftliche Einbettung, ohne die sie zu gefährlichen Instrumenten des Betriebsegoismus werden und zur Kettung der Interessenvertretung an den kapitalistischen Betrieb führen mußten, ihr Sinn also in sein Gegenteil verkehrt würde. Der spätere Streit um die Bedeutung dieser Vorschriften namentlich im Kontext der Rechtsprechung des Reichsarbeitsgerichtes bezog sich auf diesen Zusammenhang. Denn das Reichsarbeitsgericht nahm die genannten Vorschriften ernst und interpretierte sie im Sinne einer Betriebsgemeinschaft, deren Voraussetzung in den Augen der Gewerkschaften aber gerade die noch fehlende, vom Gesetzgeber zunächst vorgesehene, aber eben nicht realisierte Wirtschaftsdemokratie war. ${ }^{44}$

\section{Die Bestimmungen des Betriebsrätegesetzes}

Das Betriebsrätegesetz ${ }^{45}$ war mit über 100 Paragraphen, die sich in sechs $\mathrm{Ab}$ schnitte gliederten, ein überaus umfangreiches Gesetzeswerk. Den grundlegenden Gedanken formulierte der Paragraph 1: „Zur Wahrnehmung der gemeinsamen wirtschaftlichen Interessen der Arbeitnehmer (Arbeiter und Angestellte) dem Arbeitgeber gegenüber und zur Unterstützung des Arbeitgebers in der Erfüllung der Betriebszwecke sind in Betrieben, die in der Regel mindestens 20 Arbeiter beschäftigen, Betriebsräte zu errichten“. Bei kleineren Betrieben waren Obleute zu wählen, bei der gleichzeitigen Beschäftigung von Arbeitern und Angestellten je gesonderte Gruppenräte, die ihrerseits den Betriebsrat bildeten. Die Anzahl der Betriebsratsmitglieder variierte mit der Belegschaftszahl zwischen drei und 20; bei mehr als neun Mitgliedern mußte aus dem Betriebsrat ein vierköpfiger Betriebsausschuß gebildet werden, in dem Arbeiter und Angestellte paritätisch vertreten sein sollten. Vorsitzende und stellvertretende Vorsitzende von Betriebsrat/Betriebsausschuß vertraten den Betriebsrat rechtlich gegenüber dem Arbeitgeber sowie bei den Schlichtungs- und Arbeitsgerichtsbehörden. Das passive Wahlrecht wurde mit 24 Jahren und einjähriger Betriebszugehörigkeit erworben, das aktive

43 Wulf, Regierung, Parteien, Wirtschaftsverbände, in: Mommsen, Petzina, Weisbrod (Hg.), Industrielles System und politische Entwicklung, S. 647-657. Zum Umfeld vgl. Mommsen, Sozialpolitik im Ruhrbergbau, in: ebenda, S. 303-321.

${ }^{44}$ Flatow, Die Rechtssprechung des Reichsgerichtes, in: Neue Zeitschrift für Arbeitsrecht 6 (1926), H. 3, Sp.179-228. Nörpel, Reichsarbeitsgerichtsrechtsprechung.

${ }^{45}$ Die folgenden Ausführungen beziehen sich auf den Text des Gesetzes nach dem Stand vom $\mathrm{Fe}-$ bruar 1920, RGBI. 1920, Nr. 26, S. $146 \mathrm{ff}$. 
Wahlrecht hatten alle über 18 Jahre alten Beschäftigten des jeweiligen Betriebes. Der Arbeitgeber mußte die Wahl durch Einsetzung eines Wahlvorstandes aus den drei ältesten Arbeitnehmern einleiten, die die Wahl entsprechend der gesonderten Wahlordnung durchzuführen hatten. Kam der Arbeitgeber seinen Pflichten nicht nach, so bestand die Möglichkeit auf Antrag gegen ihn vorzugehen, der allerdings nur durch die Betriebsvertretung selbst gestellt werden konnte. In diesen Vorschriften existierte also eine Möglichkeit für Unternehmensleitungen, die Wahlen zu behindern oder zu verzögern. Erst eine Gesetzesänderung von 1928, die den Gewerkschaften das Recht auf Beantragung der Bestellung eines Wahlvorstandes durch das zuständige Arbeitsgericht einräumte, schaffte diese Verzögerungsmöglichkeit ab. ${ }^{46}$

Ungenauer waren die Bestimmungen hinsichtlich der Geschäftsführung des Betriebsrates/der Gruppenräte, die vom Gesetz nur dem Rahmen nach bestimmt war; ihre Konkretisierung hatte in den Betrieben zu erfolgen und bedurfte im Zweifelsfall der Entscheidung durch die zuständigen Schlichtungs- bzw. Arbeitsgerichtsinstanzen. Die Bestimmungen, daß Sitzungen „in der Regel und nach Möglichkeit außerhalb der Arbeitszeit" ( $(30)$ stattzufinden hatten, öffnete zukünftigen Konflikten Tür und Tor. Gleiche Wirkung mußten die Bestimmungen haben, daß die Tätigkeit als Betriebs- bzw. Gruppenrat ehrenamtlich erfolgen sollte, der Arbeitgeber aber die anfallenden Geschäftsführungskosten zu tragen hatte. Den Betriebsvertretern durfte aus ihrer Tätigkeit kein Nachteil entstehen, notwendigen Arbeitszeitausfall hatte der Arbeitgeber durch Lohnfortzahlung zu tragen $(\$ 35)$. Auch hier war der Streit um den "notwendigen Arbeitszeitausfall“ und ggf. um die Freistellung von Betriebsvertretern vorgezeichnet, da hierunter unter anderem auch jene Kosten fielen, die den Betriebsräten bei Schlichtungsbzw. Arbeitsgerichtsverfahren entstanden, so daß der Fall eintreten konnte, daß der Arbeitgeber die Kosten beider Prozeßparteien zu tragen hatte. Konflikte konnten sich auch aus den Vorschriften über die Ausstattung der Betriebsvertretungen mit den erforderlichen Unterlagen und die Zurverfügungstellung geeigneter Räume ergeben. Anpassungsprobleme in der Praxis waren schließlich in einem weiteren Bereich zu erwarten. Eigentliche Basis des Betriebsrates war der Betrieb als technisch-ökonomische Produktionseinheit. Gesamtbetriebsräte für Konzerne mit weit auseinanderliegenden Betrieben und Werken sah das Gesetz nicht vor. Wenn überhaupt konnten sie nur auf privatrechtlicher Grundlage durch freiwilligen Zusammenschluß der jeweiligen Betriebsräte gebildet werden. Eine Ausnahme existierte lediglich in dem Fall, in dem mehrere Werke eines Eigentümers räumlich eng beieinander lagen und de facto eine technische Einheit bildeten; hier konnte zwischen Einzel- oder Gesamtbetriebsräten gewählt werden: dem Taktieren beider Seiten waren mit dieser Vorschrift viele Spielräume gegeben, Konflikte daher zwangsläufig vorgezeichnet.

46 Wie erzwingt man die Errichtung eines Betriebsrates, in: Betrieb und Wirtschaft. Beilage zum "Zentralblatt der christlichen Gewerkschaften" für die Arbeitervertreter in den Betriebs- und Wirtschaftsräten, 4. Jg., Nr. 10, 15. 10. 1928. Vgl. auch Schlichtungs-Ausschuß Hamburg an Regicrungsrat Sitzler, Reichsarbeitsministerium, 8. 4. 1921 sowie Antwort des Reichsarbeitsministeriums vom 12. 4. 1921, BAP RAM, Nr. 465, Bll. 44-48. 
Die Amtszeit eines Betriebs- bzw. Gruppenrates betrug ein Jahr. Er war von der Belegschaft direkt nach einer gesondert zu erlassenden Wahlordnung zu wählen und gegen Sanktionen der Unternehmensleitungen vergleichsweise wirksam geschützt. Amtsenthebungen bedurften einer genauen Begründung aus den Vorschriften des Gesetzes oder anderer, etwa strafrechtlicher Tatbestände. Neuwahlen hatten zu erfolgen, wenn die Zahl der Betriebsvertreter eine bestimmte Zahl unterschritt und auf den Reservelisten nicht mehr genügend Nachrücker zur Verfügung standen. Diese Vorschriften stellten einen weiteren Pferdefuß des Gesetzes dar, da sie es einzelnen Fraktionen in den Betriebs- bzw. Gruppenräten ermöglichten, Neuwahlen durch Kollektivrücktritte nach Belieben zu erzwingen. Das Verhältnis der Betriebs-/Gruppenräte zur Belegschaft war durch das endgültige Gesetz völlig anders geregelt worden, als es zunächst nach dem ersten Gesetzentwurf den Anschein gehabt hatte. Nach $₫ 48$ konnte die Betriebsversammlung lediglich Anregungen geben und Wünsche äußern. Rechte irgendwelcher Art besaß sie nicht. Sie hatte außerhalb der Arbeitszeit zu tagen; der Arbeitgeber hatte entweder die nötigen Räume zu stellen oder die Anmietung von Sälen zu finanzieren: ein weiterer potentieller Konfliktpunkt. Da die Sitzungen des Betriebs- bzw. der Gruppenräte zudem nicht öffentlich waren, besaßen die Belegschaften abgesehen von ihrem Wahlrecht keine rechtlich legitimen Mittel, auf das Verhalten und Handeln der Betriebs- und Arbeiterräte Einfluß zu nehmen. Das Gesetz wollte freilich die Betriebsvertreter ohnehin nicht allein als Interessenvertreter begreifen; insofern war die Beschränkung des „Belegschaftszugriffs“ auf die Räte nur folgerichtig. Die Vorschriften des Abschnitt III ( $\$ 66 \mathrm{ff}$ ) über die Aufgaben und Befugnisse des Betriebsrates bzw. der Gruppenräte, zeigten dies an.

In die Zuständigkeit des Betriebsrates fiel

- Unterstützung der Betriebsleitung und Sorge um möglichst hohen Stand der Wirtschaftlichkeit;

- Mitwirkung bei der Einführung neuer Arbeitsmethoden;

- Wahrung des Betriebsfriedens und Anrufung des Schlichtungsausschusses bei Streitigkeiten;

- Überwachung der Durchführung rechtskräftiger Schiedssprüche;

- Vereinbarung bzw. Änderung allgemeiner Dienstvorschriften im Kontext der jeweils gültigen Tarifverträge;

- Förderung des „Einvernehmens innerhalb der Arbeitnehmerschaft sowie zwischen ihr und dem Arbeitgeber", "Wahrung der Vereinigungsfreiheit";

- Abstellen von Beschwerden der Gruppenräte;

- Mitwirkung bei der Verwaltung der Wohlfahrtseinrichtungen.

Rechte zum eigenständigen Eingriff in die Betriebsleitung besaß der Betriebsrat nicht. Das Betriebsrätegesetz gestand ihm lediglich die Einsichtnahme in die für seine Tätigkeit erforderlichen Betriebsunterlagen zu, „soweit dadurch keine Betriebs- oder Geschäftsgeheimnisse gefährdet werden“ (\$71) und verpflichtete den Arbeitgeber zur Vorlage eines vierteljährlichen Lageberichtes. Die Betriebsratsmitglieder waren ihrerseits zum Stillschweigen über betriebliche Informationen verpflichtet. Überdies hatte der Betriebsrat das Recht, bis zu zwei gleichberechtigte Vertreter in den Aufsichtsrat des jeweiligen Unternehmens zu entsenden, so- 
fern ein solcher gesellschaftsrechtlich vorgeschrieben war $(\$ 70)$, und der Arbeitgeber mußte die Bilanz sowie die Gewinn- und Verlustrechnung vorlegen und erläutern. Die beiden letzten Punkte sollten durch Ausführungsgesetze im einzelnen geregelt werden $(\$ 72)$.

Die auf diese Weise fixierten Rechte und Pflichten des Betriebsrates waren ganz offensichtlich dem „Betriebswohl“ unterworfen. Dies war weniger bedenklich als die letztlich paradoxe Vorschrift, die den Betriebsräten die Förderung der Wirtschaftlichkeit zur Aufgabe machte, andererseits aber die Geschäftsleitungen nicht zwang, ihnen die hierfür notwendigen Informationen ohne weiteres zur Verfügung zu stellen und eventuelle Ratschläge oder Eingaben der Betriebsräte zu berücksichtigen. Es mußte daher vor allem von den Ausführungsgesetzen abhängen, wie weit die wirtschaftliche Mitarbeit der Betriebsräte gesetzlich ernsthaft verankert werden sollte. Völlig anders sahen hingegen die Bestimmungen des Gesetzes über Aufgaben und Pflichten der Gruppenräte $(\$ 78)$ aus. Hier handelte es sich im wesentlichen um neun Bereiche, die sich direkt oder indirekt auf die Interessenvertretung der betroffenen Arbeitnehmergruppen richteten und daher im Gegensatz zu den Aufgaben des Betriebsrates weniger an das Betriebswohl als Referenzebene gebunden waren:

- Überwachung von Tarifverträgen und Schiedssprüchen;

- Mitwirkung bei der Regelung der Lohn- und Arbeitsverhältnisse, sofern eine tarifvertragliche Regelung nicht existiert, im Einvernehmen mit den Gewerkschaften, namentlich auch

- „bei der Festsetzung der Akkord- und Stücklohnsätze oder der für ihre Festsetzung maßgebenden Grundsätze,

- bei der Einführung neuer Löhnungsmethoden,

- bei der Festsetzung der Arbeitszeit, insbesondere bei Verlängerungen und Verkürzungen der regelmäßigen Arbeitszeit,

- bei der Regelung des Urlaubs der Arbeitnehmer und

- bei Erledigung von Beschwerden über die Ausbildung und Behandlung der Lehrlinge im Betriebe“,

- Vereinbarung der Arbeitsordnung;

- Bearbeitung von Einzelbeschwerden;

- Anrufung des Schlichtungsausschusses in Streitfällen, in denen der Betriebsrat dies ablehnt;

- Bekämpfung der Unfallgefahren;

- Vereinbarung von Einstellungsrichtlinien für die jeweilige Beschäftigtengruppe, sofern tarifvertragliche Regelungen nicht existieren. Diese Richtlinien durften keinen politisch, gewerkschaftlich, religiös, geschlechtlich oder sonstig diskriminierenden Charakter besitzen $(\$ 81)$;

- Mitwirkung bei Entlassungen. Bei Einzelentlassungen konnte der Gruppenrat begründeten Einspruch erheben, der im Falle einer Nichteinigung vor dem gesetzlichen Schlichtungsverfahren zu entscheiden war und bei einer Bejahung des Einspruchs dem Arbeitgeber die Weiterbeschäftigung oder eine angemessene Entschädigung auferlegte. Die Einspruchsgründe waren im einzelnen festgelegt (Diskriminierung, fehlende Begründung, unbillige Härte, Weigerung zur dauerhaften Ausführung nichtvereinbarter Tätigkeiten) (\$S 84-87). 
Der Gruppenrat hatte damit im Gegensatz zum Betriebsrat handfeste Mitbestimmungsrechte, die er im Zweifelsfall auch bei den zuständigen Behörden und Gerichten einklagen konnte. Ein Politikum und Kuriosum zugleich war das Kündigungsverfahren. Nicht nur gehörte der Kündigungsschutz im Prinzip nicht in die Betriebsverfassung, das arbeitsgerichtliche Verfahren wurde überdies davon abhängig gemacht, daß zuvor ein Gruppenrat einen begründeten Einspruch gegen die Kündigung erhoben hatte, den der Arbeitgeber zurückwies. Im Klartext hieß dies: Ohne Gruppenrat kein Einspruch, also auch kein im Zweifelsfall gerichtlicher Kündigungsschutz. Damit war es für zahlreiche Arbeitgeber ein einfaches Rechenexempel, ob man lieber die Strafe für die Wahlbehinderung oder die Abfindungen für ungerechtfertigte Kündigungen zahlen wollte. Das Kündigungseinspruchsrecht war ohne Frage die bedeutsamste Regelung des Betriebsrätegesetzes. Doch auch die weitgefaßten Möglichkeiten, in die Festlegung der Lohn- und Arbeitsbedingungen einzugreifen, waren von Bedeutung. Ihre Praktizierung war aber selbst Gegenstand eines betrieblichen Aushandlungsprozesses, da das Recht der Mitwirkung im Gesetz nicht präzisiert war. Zumindest waren auf diese Weise Kommunikations- und Anhörungsrechte geschaffen, im Streitfall blieb der Weg zum Schlichtungsausschuß bzw. Arbeitsgericht.

Sowohl Betriebs- als auch Gruppenräte waren im übrigen gesetzlich zur Moderation von Konflikten aufgefordert: „Bei der Wahrnehmung seiner Aufgaben hat der Betriebsrat [bzw. Gruppenrat] dahin zu wirken, daß von beiden Seiten Forderungen und Maßnahmen unterlassen werden, die das Gemeininteresse schädigen." (\$68). Seine eigenständigen Handlungsmöglichkeiten blieben indes begrenzt, da die Ausführung der gemeinsamen Beschlüsse beim Arbeitgeber lag und er nicht eigenständig in die Betriebsleitung eingreifen durfte, eine Vorschrift, die selbst wiederum zum Gegenstand innerbetrieblicher Konflikte werden mußte.

Die bisherigen einschlägigen Gesetze und Verordnungen hatten die betrieblichen Interessenvertreter vor möglichen Schikanen der Arbeitgeber nur sehr unzureichend geschützt. Das Betriebsrätegesetz brachte hier in seinem fünften Abschnitt grundlegende Neuerungen. Versetzungen oder Entlassungen waren grundsätzlich nur mit Zustimmung des Betriebsrates möglich, abgesehen von dem Fall fristloser Entlassung. Die fehlende Zustimmung zur Versetzung/Entlassung konnte durch einen Spruch im Schlichtungsverfahren auf Antrag des Arbeitgebers ersetzt werden. Ähnliches galt im übrigen im Falle der Amtsenthebung, die ebenfalls nur durch Spruch des zuständigen Schlichtungsausschusses bzw., falls ein solcher nicht existierte, durch Spruch der zuständigen Regierungsbehörde (Gewerbeaufsicht) erfolgen konnte. Im Schutz der Betriebsvertreter gab es allerdings eine Lücke. Zwar hatte der Arbeitgeber Behinderungen des Wahlverfahrens zu unterlassen, jedoch besaßen weder Mitglieder des Wahlvorstandes noch Listenkandidaten einen gesonderten Kündigungsschutz. Damit standen böswilligen Arbeitgebern weiterhin zahlreiche Möglichkeiten offen, zumindest indirekt auf die Wahlverfahren und Kandidaten Einfluß zu nehmen, und dies geschah auch - insbesondere in Klein- und Mittelbetrieben in einer Weise, die die Gewerkschaften wiederholt zu Protesten und Forderungen nach Gesetzesänderungen veranlaßte. ${ }^{47} \mathrm{Bis}$

47 Gewerbeaufsichtsberichte, Ifd. 
1933 wurde an den Schutzvorschriften der $\int \$$ 95-97 aber nichts mehr geändert. Überhaupt hielt sich die Anzahl der Initiativen, die nach 1920 zur Änderung des Betriebsrätegesetzes ergriffen wurden, in engen Grenzen, zumal das Reichsarbeitsministerium von sich aus nicht in Aktion treten wollte, da es trotz erkennbarer Funktionsdefizite zunächst die praktischen Erfahrungen mit dem Gesetz abwarten wollte. ${ }^{48}$ Lediglich die Möglichkeiten zur Wahlbehinderung durch die Unternehmer wurden 1928 durch eine Gesetzesnovelle verringert. ${ }^{49}$

Das Betriebsrätegesetz, obwohl im Nachhinein gelegentlich so hingestellt, war seinen inhaltlichen Bestimmungen nach kein Kompromiß. Inhaltlich war es auch nicht der Versuch, wie es vor allem Anhänger des Rätegedankens insinuierten, die proletarische Radikalität durch Sozialpolitik zu kanalisieren. Zweifellos spielte der Pazifizierungsgedanke bei der MSPD und der Mehrheit der freien Gewerkschaften 1919 eine gewisse Rolle, doch war dies entscheidend für den Zeitpunkt der Verabschiedung des Gesetzes, weniger für seinen Inhalt. Auf den hatte die sozialdemokratische Behördenspitze (Reichsarbeitsminister Bauer, Reichsarbeitsminister Schlicke) ohnehin nur geringen Einfluß. Die konzeptionelle Arbeit lag bei jenen Beamten, die sich in der sozialpolitischen Tradition der Vorkriegszeit als Träger einer vorsichtigen Sozialreform begriffen..$^{50}$ Dies war auch deshalb möglich, weil weder die MSPD noch die Mehrheit der freien Gewerkschaften ein ernsthaftes Interesse am Betrieb hatten. Arbeiterausschüsse/Betriebsräte waren ungefährlich, teilweise vielleicht nützlich, solange sie nicht in den eigentlichen Arbeitsbereich der Gewerkschaften eindrangen. Der betriebliche Arbeitsalltag war den meisten führenden Freigewerkschaftern herzlich gleichgültig ${ }^{51}$, im Unterschied zu den christlichen Gewerkschaften, die das Betriebsrätegesetz folgerichtig sehr ernst nahmen. Das Gesetz ging - wie die bürgerliche Sozialreform - vom Gedanken der Betriebseinheit bzw. des Betriebswohls aus, in der/dem Effizienz und Interessenvertretung bei Aufrechterhaltung der grundlegenden, durch Eigentum legitimierten Machtasymmetrie miteinander zu kombinieren waren. Der Betriebsrat/Gruppenrat erhielt eine doppelte Referenzebene: Betriebswohl und Gruppeninteressen. Die Aufspaltung in eine duale Struktur von Betriebsräten mit der Referenzebene Betriebswohl und Gruppenräten mit der Referenzebene Belegschafts-/Gruppeninteressen und deren Vertretung war zwar nicht beabsichtigt, folgte aber aus einem Gesetzestext, der aus politischen Gründen an einer getrenn-

48 Siehe dic Zusammenstellung von Änderungsanträgen und Gesetzesinitiativen in: BAP RAM Nr. 500, 501, RJM Nr. 2186.

49 Anträge auf Verbesserung des Betriebsrätegesetzes im Reichstag, in: Gewerkschaftszeitung 1927, Nr. 11, S. 144 f. Kalckbrenner, Das Abänderungsgesetz zum Betriebsrätegesetz, in: RABl. 1928, II, S. $95 \mathrm{f}$.

50 Sitzler, Das Werden der Betriebsdemokratie, in: RABl. 1928, II, S. 204 f. Neben Sitzler als dem zuständigen Abteilungsleiter waren von besonderer Bedeutung der Regierungsrat Johannes Feig und der Hilfsreferent Georg Flatow. Letzterer stand der SPD nahe und wechselte später zum Preußischen Ministerium für Handel und Gewerbe. Von diesen federführenden Beamten stammten auch die zwei meist zitierten Gesetzeskommentare. Feig, Sitzler, Das Betriebsrätegesetz. Flatow, Kommentar zum Betriebsrätegesetz. Vgl. auch Dersch, Kommentar zum Betriebsrätegesetz. Der Kommentar von Feig und Sitzler war zugleich ein Dokument des etatistisch-sozialreformerischen Selbstverständnisses im Reichsarbeitsministerium.

51 Die von Oertzen im einzelnen nachgezeichneten Debatten um dic Räte-Konzeption, insbesondere die für die MSPD vergleichsweise repräsentative Auffassung von Hugo Sinzheimer, zeigen die z.T. flagrante Unkenntnis der betrieblichen Probleme. 
ten Behandlung von Arbeitern und Angestellten festhielt. Diese Unterscheidung war in ihrer Wirkung erheblich, da sie die Kommunikation der beteiligten Gruppen im Betrieb in hohem Maße strukturierte und zu zahlreichen Mißverständnissen Anlaß gab.

Neben der Trennung zwischen gemeinwohlverpflichtetem Betriebsrat und interessenbezogenen Gruppenräten ergaben sich die meisten „offenen Fragen“ zunächst aus der Konkretisierung der „Geschäftsführung“, an zweiter Stelle aus der Interpretierbarkeit der im Gesetz vorgesehenen Mitwirkungs- und Mitbestimmungsrechte. Bezog sich das erste auf die allgemeine Handlungsfähigkeit der Betriebsvertretungen, so betraf das zweite die Präzisierung von Rechten und Verfahrensstrukturen im Einzelfall. Das Gesetz wäre aber selbst bei präziserer Formulierung von Vorschriften nicht dazu in der Lage gewesen, seine eigene Praktizierung bis ins Einzelne vorzugeben. 1930 bemerkte Ernst Fraenkel: „Das Betriebsräterecht von 1930 ist etwas anderes als das Betriebsrätegesetz von 1920, trotzdem der Wortlaut des Gesetzestextes im wesentlichen unverändert geblieben ist. ... Die Normen des Gesetzes haben ... in Verfolg der sozialen und wirtschaftlichen Entwicklung eine Eigenbewegung erhalten.... Die Geschichte eines Gesetzes ist mit dem Augenblick seiner staatsrechtlichen Sanktionierung nicht abgeschlossen, sondern erst in ihr entscheidendes Stadium getreten. ${ }^{\text {52 }}$

Der Gesetzgebungsprozeß war im Januar 1920 aber auch ganz formal noch nicht beendet. Das Gesetz schrieb die Verabschiedung von zumindest zwei Ausführungsgesetzen vor. Zunächst galt es das Recht der Betriebsräte, in bestimmte Geschäftsunterlagen Einsicht zu nehmen, zu regeln, daraufhin deren Recht, Vertreter in die Aufsichtsräte der entsprechenden privatwirtschaftlichen Gesellschaften zu entsenden. In der Reichsregierung einigte man sich darauf, daß für die Ausarbeitung des Aufsichtsratsgesetzes das Reichsarbeitsministerium, für das Betriebsbilanzgesetz aber das Reichsjustizministerium federführend sein sollte, das im übrigen „in Anbetracht der bedeutsamen Einwirkungen der Bestimmungen eines derartigen Gesetzes auf das geltende Handelsrecht" auch bei dem Aufsichtsratsgesetz ein entscheidendes Wort mitreden wollte. ${ }^{53}$ Die Frage der Vorlage der Betriebsbilanz und anderer Geschäftsunterlagen hatte bereits bei der Diskussion des Gesetzentwurfes im Sozialpolitischen Ausschuß der Nationalversammlung eine Rolle gespielt. Schon im Herbst 1919 hatten sich die Industrievertreter recht erfolgreich darum bemüht, den Begriff der Betriebsbilanz im $\$ 72$ des BRG derart unscharf fassen zu lassen, daß insbesondere durch eine eigentümliche Trennung von Geschäfts- und Privatvermögen, wobei über letzteres keine Auskunft gegeben zu werden brauchte, die Informationspflicht der Unternehmensleitungen minimiert wurde. Doch genügte dies offensichtlich nicht. Unmittelbar im Anschluß an die Verabschiedung des BRG, das ein Ausführungsgesetz zum $₫ 72$ bis zum Jahresende 1920 verlangte, setzte der RDI unter Mitarbeit des Kölner Betriebswirtes Eugen Schmalenbach eine Kommission ein, die zum 1. September 1920

52 Fraenkel, Zehn Jahre Betriebsrätegesetz, in: Die Gesellschaft 7 (1930), S. 117.

53 Staatssekretär Joel, Reichsjustizministerium, an den Reichsarbeitsminister, 11. 3. 1920, BAP RAM 489, Bl. 12. 
einen eigenen Gesetzentwurf vorlegte. Nach diesem Gesetzentwurf hatte die Unternehmensleitung lediglich nichtssagende Gesamtzahlen vorzulegen. ${ }^{54}$

Das Reichsjustizministerium schloß sich diesem Vorschlag zwar nicht an, legte aber bei der ersten Besprechung mit den verschiedenen Interessengruppen am 22. September 1920 von sich aus keinen eigenen Entwurf, sondern lediglich Rahmenüberlegungen vor, die weitgehend dem Diskussionsstand im Vorfeld der Verabschiedung des Betriebsrätegesetzes entsprachen. Die Interessenvertreter waren sich schnell einig, die vorlagepflichtige Bilanz auf die Vorschriften des Handelsgesetzbuches zu beziehen. Auch in der Frage, ob den Betriebsräten, die häufig nur Einzelbetriebe größerer wirtschaftlicher Unternehmen vertraten, Teil- oder Gesamtbilanzen vorzulegen seien, war man sich im Prinzip einig, daß nur die Vorlage von Gesamtbilanzen sinnvoll sei. Die Auseinandersetzungen blieben erstaunlich unspektakulär. Lediglich der Gewerkschaftsbund der Angestellten verlangte eine Ausdehnung der Auskunftspflicht auch auf Bilanzunterlagen, ohne sich mit dieser Forderung allerdings durchsetzen zu können. ${ }^{55}$ Der Regierungsentwurf lag angesichts dieser Situation schnell vor ${ }^{56}$, der Reichstag stimmte am 3. Februar 1921 zu. ${ }^{57}$ Nach dem Gesetz, das am 5. Februar 1921 in Kraft trat, hatten die Betriebsräte das Recht, sich die Bilanz sowie die Gewinn- und Verlustrechnung vorlegen und erläutern zu lassen. Weitergehende Informationsrechte, insbesondere eine Einsicht in Bilanzunterlagen, waren nicht vorgesehen, so daß Betriebsausschuß/ Betriebsrat auf das Wohlwollen der Unternehmensleitungen bei der Informationsweitergabe angewiesen waren, da die dürren Bilanzzahlen ohne detaillierte Erläuterung nicht aussagefähig waren. Die Unternehmensleitungen waren im übrigen nicht einmal verpflichtet, die Bilanzen und die G-V-Rechnungen in schriftlicher Form vorzulegen. Formal genügte es, wenn dem Betriebsrat die Zahlen vorgelesen wurden. Es sollte späterhin sogar zu Prozessen um die Frage kommen, ob die Betriebsräte sich Aufzeichnungen über die Bilanz machen durften. Wilhelm Kalveram resümierte die Gesetzeslage 1925 daher zutreffenderweise folgendermaßen: „Das Betriebsbilanzgesetz als Ausweitung der Rahmenvorschrift des Betriebsrätegesetzes hat die Erwartungen der Arbeitnehmerschaft nicht erfüllt. Man darf wohl sagen, daß dem Betriebsrat da, wo die Geschäftsleitung bei ihren Bilanzvorlagen und Auskünften die durch das Gesetz gezogenen Grenzen nicht überschreitet, ein klarer Einblick in die Produktions-, Absatz-, Kapital- und Rentabilitätsverhältnisse nicht gelingen wird. ${ }^{~} 58 \mathrm{Da}$ dem Betriebsrat eine Überprüfung der vorgelegten Zahlen faktisch unmöglich war - einerseits hatte er über sie Stillschweigen zu wahren, andererseits durfte er von sich aus keine Überprüfung der Zahlen einleiten ${ }^{59}$-, war das Betriebsbilanzgesetz ohne die Kooperation der Unternehmensleitungen funktionsunfähig. Der Deutsche Werkmeister-Verband

54 RDI an Reichsarbeitsministerium, 1. 9. 1920, BAP RAM 497, Bll. 34-36.

55 Aufzeichnung über die Besprechung vom 2. September, betreffend die Gestaltung des im $\$ 72$ des BRG vorbehaltenen Gesetzes über die Betriebsbilanz und die Betriebs-Gewinn- und Verlustrechnung, BAP RAM 497, Bll. 75-79.

56 Siehe die Entwürfe im einzelnen in: BAP RJM 2187, 2188.

57 Reichstagspräsident Löbe an RJM, 4. 2. 1921, BAP RAM 469, Bl. 195. Gesetzestext in: RGBl. 1, 1921, S. 159.

58 Kalveram, Bilanzprobleme, in: Potthoff (Hg.), Soziale Probleme des Betriebes, S. 47.

59 RJM an RAM, 21. 5. 1921, BAP 498, Bll. 31-32. 
gab sich am 18.Februar 1921 denn auch keinerlei Illusionen bei der Beurteilung des Gesetzes hin: Das neue Gesetz besitze „eigentlich nur einen gewißen Papierwert..." Mit ihm sei „weder den Interessen der Arbeitnehmer, noch der Allgemeinheit ... gedient... Es ist einmal wieder ein Gesetz geschaffen, welches nach außen hin allerdings einen sozialpolitischen Einschlag vortäuscht, innerlich aber jeder Sozialpolitik bar ist, und dem Unternehmertum die Freiheit läßt, die es immer besessen hat. Unsere Gesetzsammlung ist um ein Imaginär-Produkt vermehrt, aber innerlich ist das deutsche Volk damit nicht reicher geworden. “60

Im September 1920 legte das Reichsarbeitsministerium einen ersten Gesetzentwurf zur Aufsichtsratsmitbestimmung vor. Nach der Billigung des Entwurfes durch die Vertreter der Länder ${ }^{61}$, lud das Reichsarbeitsministerium am 16. Oktober 1920 zu einer Besprechung der Spitzenverbände der Wirtschaft und der Gewerkschaften, auf der sehr rasch die Bedenken der Arbeitgeberseite deutlich wurden. Im Arbeitgeberlager befürchtete man, daß bei einer vollständigen Gleichberechtigung der Arbeitnehmervertreter mit den Eigentümerrepräsentanten im Aufsichtsrat dessen Arbeitsfähigkeit leide und eine derartige Klausel, wie sie der Gesetzentwurf vorsah, überdies die Vorschriften des einschlägigen $\$ 70$ des BRG überschreite, der eine Vertretung der Betriebsräte im Aufsichtsrat nur zum Zwecke der Vertretung der sozialen Belange der Arbeitnehmer zulasse. ${ }^{62}$ Das Reichsarbeitsministerium und mit ihm die Mehrheit der vertretenen Gewerkschaften erblickten in der Formulierung des $\$ 70$ BRG (Betriebsräte werden in den Aufsichtsrat entsandt, „um die Interessen und Forderungen der Arbeitnehmer sowie deren Wünsche hinsichtlich der Organisation des Betriebes zu vertreten") allerdings lediglich den Anlaß zur Entsendung von ansonsten gleichberechtigten Aufsichtsratsmitgliedern aus den Betriebsräten. Für das Reichsarbeitsministerium und seine federführenden Beamten war die gleichberechtigte Vertretung der Betriebsräte im Aufsichtsrat die Einlösung eines Versprechens, das im Kontext der Entstehung des Betriebsrätegesetzes den Arbeitern gemacht worden war. In der Begründung des überarbeiteten Gesetzentwurfes zitierte man die Begründung des BRG und bezeichnete die Aufsichtsratsvertretung „als eine ,Neuerung völlig grundlegender Art', als Verwandlung des im ,allgemeinen gewährten Mitberatungsrechts in ein Mitbestimmungsrecht ${ }^{\star}$ das vorgeschlagen werde, in der Überzeugung, daß nichts so sehr die Arbeitsfreudigkeit, das Verantwortlichkeitsgefühl und das Interesse an der Hebung der Betriebsleistungen und des Ertrages zu steigern geeignet ist, als die verantwortliche Mitwirkung an der obersten Leitung des Unternehmens, daß solche jeder äußerlichen Kontrolle bei weitem überlegen ... ist. " ${ }^{63}$ Man berief sich zugleich auf die Verhandlungen des sozialpolitischen Ausschusses der Nationalversammlung. „Es habe kein Zweifel darüber bestanden“, so der neue Reichsarbeitsminister Brauns (Zentrum) im Dezember 1920, „daß die Arbeitnehmermitglieder des Aufsichtsrates die gleichen Rechte und Pflichte wie

60 Deutsche Werkmeister-Zeitung, 38. Jg., Nr. 7, 18. 2. 1921.

61 Protokoll der Sitzung vom 29. 9. 1920, BAP, RAM 489, Bll. 82-86.

62 Protokoll der Besprechung vom 29. 10. 1920, BAP RAM 489, Bll. 186-194. Niederschrift über das Ergebnis der am 8.11. 1920 im Reichsarbeitsministerium abgehaltenen Besprechung des vorläufigen Referentenentwurfs eines Gesetzes zur Ausführung des $\$ 70$ des BRG; BAP RAM 489, Bll. 195-203.

63 Überarbeiteter Gesetzentwurf mit Begründung, BAP RAM 489, Bll. 240-245. 
die übrigen Aufsichtsratsmitglieder haben sollten. ... Man müsse sich eben vergegenwärtigen, daß wir zwar einerseits noch auf dem Boden der privatkapitalistischen Wirtschaft ständen, daß aber $\$ 70$ eine gewisse Sozialisierung bedeute." Im übrigen sei auch die Entstehungsgeschichte des BRG zu berücksichtigen: „Infolge der kritischen Lage bei der Beratung des Betriebsrätegesetzes, die befürchten ließ, daß der ganze Entwurf des Gesetzes an dem $\$ 70$ scheiterte, hätten zur Vermeidung einer parlamentarischen Krise Verhandlungen bei dem Reichskanzler stattgefunden, deren Kern die Verleihung gleichberechtigter Rechte und Pflichten an die Betriebsratsmitglieder im Aufsichtsrat gewesen sei. Deshalb könne die heutige Regierung von dem Standpunkte der Gleichberechtigung nicht abgehen, um so weniger als die Bestimmung des $\$ 70$ eine politische Konzession an die Arbeiter gewesen sei, um den Gesetzentwurf über die politischen Schwierigkeiten hinwegzubringen." ${ }^{64}$

Die Proteste der organisierten Wirtschaft blieben angesichts dieser eindeutigen Haltung des Reichsarbeitsministeriums zumindest bei den Arbeitsbehörden ohne Wirkung; bei anderen Regierungsstellen waren die Interventionen, die der Zentralausschuß der Unternehmerverbände, i.e. ein Zusammenschluß der wirtschaftlichen Spitzenverbände, am 6. Januar 1921 in einer Eingabe an den Reichsrat noch einmal zusammenfaßte und in einem eigenen Gesetzentwurf bündelte ${ }^{65}$, offensichtlich erfolgreicher. Als im Reichskabinett am 15. Januar 1921 der Entwurf beraten wurde, geriet jener $₫ 3$ des Gesetzes, der die Gleichberechtigung der Betriebsräte im Aufsichtsrat festschrieb, in die Kritik von Reichsschatz- und Reichswirtschaftsministerium, die beide für seine Streichung plädierten. Das Stimmungsbild im Kabinett war ausgeglichen. Lediglich die Stimme des Reichskanzlers gab schließlich den Ausschlag zur Annahme des Gesetzentwurfes. ${ }^{66}$ Während der Entwurf danach seine parlamentarischen Hürden zu nehmen hatte, wobei die Fragen der Gleichberechtigung und der Haftung der Aufsichtsratsmitglieder aus den Betriebsräten - letztere Frage war von den freien Gewerkschaften aufgeworfen worden, die einen Haftungsausschluß forderten - im Mittelpunkt der Debatten standen, begannen die verschiedenen wirtschaftlichen Interessenverbände eine breit angelegte Kampagne, deren Ziel die Berliner Ministerien, parlamentarischen Körperschaften und Parlamentarier waren, denen die negativen Folgen einer gleichberechtigten Teilhabe von Arbeitnehmern an den Aufsichtsräten in den düstersten Farben gemalt wurden. Nachdem am 21. Februar 1921 der sozialpolitische Ausschuß des vorläufigen Reichswirtschaftsrates mit geringfügigen Änderungswünschen zugestimmt hatte ${ }^{67}$, kam es im Vorfeld der Behandlung des Gesetzentwurfes im sozialpolitischen Ausschuß des Reichstages am 3. März 1921 noch einmal zu einer umfassenden Eingabe des DIHT. ${ }^{68}$ Diese Eingaben verfehlten ihre Wirkung nicht. Der Reichstagspräsident übersandte sie am 20. April 1921 an das Reichsarbeitsministerium mit der Bitte, man möge sie bei der weiteren Ar-

${ }^{64}$ Niederschrift über das Ergebnis der am 1. Dezember 1920 im Reichsarbeitsministerium abgehaltenen Besprechung des vorläufigen Referentenentwurfs eines Gesetzes zur Ausführung des $\$ 70$ BRG; BAP RAM 489, Bll. 285-291.

65 Zentralausschuß der Unternehmerverbände an Reichsrat, 6. 1. 1921, BAP RAM 489, Bl. 303-307.

66 Auszug aus dem Protokoll des Reichsministeriums, 15. 1. 1921, BAP RAM 489, Bl. 302.

67 BAP RAM 489, Bl. 352.

68 24. 2. 1921, BAP RAM 389, Bl. 354. 
beit am Gesetzentwurf berücksichtigen.$^{69}$ Erst im Juni 1921 stimmte der vorläufige Reichswirtschaftsrat endgültig zu; danach wurde der Entwurf dem Reichsrat zur Prüfung der Verfassungsmäßigkeit vorgelegt. ${ }^{70}$ Der Reichsrat entschied über das Aufsichtsratsgesetz allerdings erst am 5 . November $1921 .^{71}$

Die Verabschiedung des Gesetzes - eigentlich hatte es zum 1. Januar 1921 in Kraft treten sollen - verzögerte sich auf diese Weise mehr und mehr. Auf Seiten der Arbeitgeber wurde diese Zeit genutzt, um, da man schon die Gesetzesvorschriften nicht entscheidend ändern konnte, zumindest ihr praktisches Unterlaufen vorzubereiten. Ließ sich die gleichberechtigte Vertretung der Betriebsräte im Plenum des Aufsichtsrates nicht verhindern, empfahl ein Leipziger Syndikus den betroffenen Gesellschaften, so solle man die Satzungen in einer Weise ändern, daß wichtige Entscheidungen in AR-Ausschüssen getroffen würden, zu denen die Betriebsräte keinen Zugang hätten. Der Gewerkschaftsbund der Angestellten, der im übrigen als einzige Organisation während der Gesetzgebungsphase auf eine Ausweitung der Mitbestimmungsvorschriften im Sinne einer paritätischen Besetzung der Aufsichtsräte gedrungen hatte, verlangte daraufhin, gegen derartige Praktiken Sicherungen in das Gesetz aufzunehmen, scheiterte damit jedoch an der gemeinsamen Auffassung von Reichsarbeits- und Reichsjustizministerium, die nicht in die Satzungshoheit der Gesellschaften eingreifen wollten. ${ }^{72}$ Als der Reichsarbeitsminister mit Schreiben vom 30. November 1921 den Gesetzentwurf endgültig in den Reichstag einbrachte, hatte sich der Wortlaut im Vergleich zu den ersten Entwürfen nicht mehr sehr geändert: Lediglich die Bindung der Aufsichtsratsmitglieder an die Bestimmungen des BRG, Amtsdauer etc., waren noch deutlicher gefaßt. Der Reichstag verabschiedete das Gesetz am 1. Februar 1922: nunmehr hatten die Betriebsräte das Recht, aus ihrer Mitte in die Aufsichtsräte von Aktiengesellschaften, bergrechtlichen Gesellschaften und GmbH's mit Aufsichtsoder Verwaltungsrat zwei Mitglieder für den Fall zu wählen, daß dem Gremium ohnehin drei oder mehr Mitglieder angehörten, ansonsten konnte ein Betriebsrat entsandt werden. ${ }^{73}$ Die Mitglieder aus dem Betriebsrat waren den anderen Aufsichtsratsmitgliedern gleichgestellt; sie unterlagen damit auch den allgemeinen Haftungsbestimmungen. Gegen die frühzeitig erkennbare Tendenz, das Gesetz durch Satzungsänderungen ins Leere laufen zu lassen, insbesondere die Betriebsräte nicht an Personaldebatten und Entscheidungen über die Einkommen der leitenden Angestellten teilnehmen zu lassen, hatte der Gesetzgeber keine Vorsorge getroffen. Es waren daher starke Konflikte bei der Realisierung der gesetzlichen Vorschriften zu erwarten, zumal so mancher Industrielle frühzeitig signalisierte, in der Frage der Aufsichtsratsvertretung in der Praxis nicht entgegenkommend sein zu wollen. ${ }^{74}$

69 Reichstagspräsident Löbe an Reichsregierung, 20.4.21, BAP RAM 490, Bll. 48-61.

70 Schreiben des RAM, 23. 7. 1921, BAP RAM 490, Bl. 219.

71 Vermerk Dr. Flatow, BAP RAM 490, Bl. 236.

$72 \mathrm{GdA}$ an Reichsarbeitsministerium, 11.4. 1921, BAP RAM 490, Bll. 67-69. Reichsjustizministerium an Reichsarbeitsministerium, 23. 4. 1921, BAP RAM 490, Bll. 71-72.

73 Aktennotiz RAM o.D., BAP RAM 490, Bl. 348.

74 Entsendung von Betriebsratsmitgliedern in den Aufsichtsrat, RDI-Mitteilungen 1922, Nr. 384, S. $218 \mathrm{f}$. Die spätere Praxis bestätigte diese Haltung der Arbeitgeberschaft weitgehend. Von wirksamer Aufsichtsratsmitbestimmung konnte auch dann nicht gesprochen werden, wenn die Unter- 
Diese geringe Kooperationsbereitschaft war zugleich Ausdruck für die Tatsache, daß sich ähnlich wie beim Betriebsrätegesetz der sozialreformerische Ansatz des Reichsarbeitsministeriums erneut gegen die Vorstellungen der organisierten Industrie durchgesetzt hatte, auch wenn 1921 bereits der politische Druck fehlte, um zu einer schnellen Verabschiedung des Gesetzesvorhabens zu kommen. Die Praktizierung des Betriebsrätegesetzes und seiner Ausführungsgesetze stand also unter der Hypothek, daß diese Vorschriften gegen den Willen der Arbeitgeber zustande gekommen waren, was deshalb erhebliche Konsequenzen haben mußte, da es sich um Verfahrensgesetze handelte, in denen die Verfahren selbst zunächst nur dem Rahmen nach fixiert waren. Die Anwendung dieser Vorschriften und damit die erfolgreiche Praktizierung der neuen Betriebsverfassung hing nicht zuletzt aber von der Bereitschaft ab, die gesetzlichen Verfahren betriebsnah zur Regelung materieller Interessenkonflikte zu nutzen, d. h. aber vor allem, sie zunächst zu akzeptieren und in betriebliche Kommunikations- und Handlungsstrukturen zu übersetzen. In materieller Hinsicht definierte die neue Betriebsverfassung Mitbestimmungsrechte im sozialen, Mitwirkungsrechte im wirtschaftlichen Bereich, wobei die gleichberechtigte Aufsichtsratsvertretung zugleich den Einstieg in die wirtschaftliche Mitbestimmung auf Unternehmensebene brachte. Die materiellen Mitbestimmungsrechte bezogen sich im sozialen Bereich zwar explizit nur auf die Interessenvertretung der Belegschaft bzw. einzelner Belegschaftsteile. Da sie aber damit zugleich den Kern der Arbeitsorganisation betrafen (Akkorde, Lohnsysteme allgemein, Entlassungen, Arbeits- und Dienstordnung etc.), war ihre betriebswirtschaftliche Wirksamkeit potentiell erheblich größer als es in der Betonung der „nur“ sozialen Mitbestimmungsrechte zum Ausdruck kommt. Denn wäre das Betriebsrätegesetz in der Tat jene Chimäre gewesen, für die es zahlreiche deutsche „Revolutionäre“ hielten, wäre der Widerstand zahlreicher Unternehmer kaum derart massiv gewesen.

Initiativen zur Änderung des BRG in den folgenden Jahren blieben gering. Das Reichsarbeitsministerium plädierte mit guten Gründen für eine längere Erprobungsphase des Gesetzes, bevor es eine Novellierung ins Auge fassen wollte. Die Unternehmer begriffen relativ schnell, daß der Gesetzestext ihnen hinreichende Möglichkeiten betriebsnaher Anwendungen ließ und sich die Betriebsräte nicht notwendig als Nachteil herausstellten ${ }^{75}$, die freien und christlichen Gewerkschaften schließlich konnten mit dem BRG hoch zufrieden sein. Novellierungsanträge

nehmen nicht versuchten, den Einfluß der Betriebsratsmitglieder künstlich zu begrenzen. Vgl. Hans-Böckler-Stiftung (Hg.), Die Praxis der Weimarer Betriebsräte im Aufsichtsrat. In dieser Sammlung zeitgenössischer Stellungnahmen kommen vor allem Gewerkschaftsvertreter zu Wort, die die Aufsichtsratsmitbestimmung pessimistisch beurteilten. Ausgewogener waren die Berichte der Enquete-Kommission, vgl. Ausschuß zur Untersuchung der Erzeugungs- und Absatzbedingungen der deutschen Wirtschaft. Verhandlungen und Berichte des Unterausschusses für allgemeine Wirtschaftsstruktur (I. Unterausschuß), 3. Arbeitsgruppe: Wandlungen in den wirtschaftlichen Organisationsformen: Erster Teil, Wandlungen in den Rechtsformen der Einzelunternehmungen und Konzerne, Berlin 1928; Dritter Teil: Wandlungen in der aktienrechtlichen Gestaltung der Einzelunternehmen und Konzerne - Generalbericht, Berlin 1930.

75 Gleichwohl gab es auch immer wieder generelle Angriffe auf das kollektive Arbeitsrecht und die partizipativ orientierte Betriebsverfassung. So verlangte anläßlich seiner Jahrestagung der Deutsche Industrieschutzverband am 17.7.1925 die Beseitigung des kollektiven Arbeitsrechts, BAP RAM 500, Bl. 324. 
größerer Reichweite kamen daher fast ausschließlich entweder von linksradikaler Seite oder aus dem Spektrum der Angestelltenorganisationen, namentlich von dem Gewerkschaftsbund der Angestellten und dem Deutschen Werkmeister-Verband. ${ }^{76}$ Die Mehrzahl der Novellierungsforderungen bezog sich auf die Amtsdauer der Betriebsräte, die Wahlverfahren, die Sicherung der Funktion der Betriebsräte im Aufsichtsrat, das Kündigungsschutzrecht insbesondere älterer Arbeitnehmer und auf jene Paragraphen, die die Betriebsräte vor Schikanen schützen sollten. ${ }^{77}$

Die einzige größere, von Erfolg gekrönte Initiative war die bereits erwähnte Novellierung des BRG von 1928, die den Unternehmensleitungen die Möglichkeit zur Wahlbehinderung durch Unterlassung der Einsetzung eines Wahlvorstandes aus der Hand nahm. Hintergrund war der starke Rückgang von Betriebsvertretungen nach $1924^{78}$ und das erkennbare Interesse mancher Arbeitgeber, durch Wahlbehinderung das Kündigungsschutzrecht auszuhebeln. ${ }^{79}$ Das Reichsarbeitsministerium war auch hier zunächst gegenüber Gesetzesinitiativen zurückhaltend, zumal der zuständige Regierungsrat Friedrich Sitzler glaubte, die bestehenden Möglichkeiten, die Gewerbeaufsicht einzuschalten und von Belegschaftsversammlungen aus Strafanträge gegen unwillige Arbeitgeber zu stellen, seien hinreichend. Wiederkehrende Reichstagsentschließungen wurden daher zurückgewiesen.$^{80}$ Die Klagen rissen jedoch nicht ab und blieben auch keineswegs auf das Umfeld der freien Gewerkschaften beschränkt. Anfang 1927 schlossen sich auch der Deutschnationale Handlungsgehilfenverband und der Deutsche Gewerkschaftsbund den Novellierungsforderungen $a^{81}$, die SPD brachte im Februar einen Entschließungsantrag im Reichstag zur Änderung des BRG ein ${ }^{82}$, weitere Anträge des Zentrums, der Kommunisten und Nationalsozialisten folgten. Der Antrag der SPD verlangte eine Novellierung des Wahlparagraphen 23 des BRG dahingehend, daß eine Bestellung des Wahlvorstandes auch durch die Belegschaftsversammlung möglich würde. Das Reichsarbeitsministerium war aber weiterhin nicht bereit, einen eigenständigen Gesetzentwurf vorzulegen, sondern überließ die Arbeit dem zuständigen Reichstagsausschuß ${ }^{83}$, da man, wie Friedrich Sitzler dem Minister als Antwort auf den SPD-Vorschlag empfahl, eine Abänderung des BRG grundsätzlich für verfrüht hielt, „da das Gesetz sich bisher lediglich in einer Periode wirtschaftlicher und politischer Krisen hat auswirken können." 84 Schließlich erklärte sich der Reichsarbeitsminister mit dem Novellierungsvor-

76 Siehe vor allem Deutscher Werkmeister-Verband, Denkschrift zur Frage des Ausbaus des Betriebsrätegesetzes, 2. 9. 1929, BAP RJM (30.01) 2186, Bll. 55-65.

77 Im einzelnen BAP RAM 500, 501, 502. RJM 2186.

78 Zur Betriebsrätekrise vgl. Werner Plumpe, Die Betriebsräte in der Weimarer Republik, S. 42-60.

79 Siehe die Ausarbeitung von Peter Funk, Warum so viele Betriebe ohne Betriebsvertretung? (1926), BAP RAM 501, Bll. $39 \mathrm{f}$.

${ }^{80}$ Vorlage Sitzler für den Reichsarbeitsminister, 19. 2. 1926, BAP RAM 501, Bl. 38.

81 DHV Schlesien an Reichsarbeitsministerium, 10.5. 1927, BAP RAM 501, Bl. 54. Deutscher Gewerkschaftsbund an die dem DGB nahestehenden Mitglieder der Reichstagsfraktionen, 16.3. 1927, BAP RAM 501, Bll. 167-169.

82 Reichstagsdrucksachen 3. Wahlperiode 1924/27, Nr. 3049.

83 Vorlage für den Reichsarbeitsminister, Ministerentscheid o.D., BAP RAM 501, Bll. 62, 64

84 Vorlage Dr. Sitzler für den Reichsarbeitsminister, 26. 2. 1927, BAP RAM 501, Bl. 166. 
schlag der SPD einverstanden. ${ }^{85}$ Im zuständigen sozialpolitischen Ausschuß des Reichstages forderten im Dezember die Vertreter der SPD nunmehr allerdings eine zusätzliche Änderung des $\$ 23$ BRG, die neben den Belegschaften auch den im Betrieb vertretenen Gewerkschaften ein Recht zur Anrufung des Arbeitsgerichtes, das bei Weigerung des Arbeitgebers den Wahlvorstand einzusetzen hatte, einräumte. ${ }^{86} \mathrm{Obwohl} \mathrm{die} \mathrm{Reichsregierung} \mathrm{in} \mathrm{einer} \mathrm{Regierungserklärung} \mathrm{am} \mathrm{31.} \mathrm{Ja-}$ nuar 1928 vor dem sozialpolitischen Ausschuß des Reichstages dieses Recht verneinte, „da die Durchführung des Betriebsrätegesetzes ihrer Art nach mehr eine Aufgabe der staatlichen Aufsicht als der Initiative der Beteiligten selbst sei ${ }^{\text {“ }}{ }^{7}$, gab sie der Initiativgesetzgebung des Reichstages einen Tag später nach, als sie merkte, daß hierfür eine klare Mehrheit existierte. ${ }^{88}$ Am 1. Februar 1928 nahm der sozialpolitische Ausschuß des Reichstages einen entsprechenden Gesetzentwurf gegen die Stimmen von DVP und DNVP an ${ }^{89}$ Der Reichstag schloß sich an; am 28. Februar 1928 trat das Gesetz in Kraft. ${ }^{90} \mathrm{Daß}$ die Arbeitgeberverbände das Gesetz nicht begrüßten, sondern den Betrieb zunehmend im Würgegriff der Gewerkschaften wähnten, bedarf keiner weiteren Ausführungen. ${ }^{91}$

Der Gesetzestext, so wie er 1919/20 festgelegt und in wenigen Ausführungsgesetzen präzisiert worden war, blieb während der gesamten Zeit der Weimarer Republik in Kraft. Niemand hatte ein erkennbares Interesse, ihn grundlegend zu ändern oder gar zu beseitigen, von einigen eher abseitigen Initiativen aus der mittelständischen Industrie abgesehen. Selbst die Schwerindustrie unternahm keine Anläufe, den Gesetzestext zu ändern, auch wenn ihre Kritik an der Praxis der Betriebsräte grundsätzlich blieb. Die Beseitigung der Weimarer Betriebsverfassung 1933/34 stellte insofern einen grundlegenden Bruch dar, der zudem nicht unmittelbar erfolgte, sondern eine gewisse Zeit beanspruchte, in der das Betriebsrätegesetz noch weiterhin in Kraft blieb.

\section{Die Beseitigung des Betriebsrätegesetzes}

1931 fanden die letzten Betriebsratswahlen in der Weimarer Republik statt. 1932 wurden sie u. a. wegen befürchteter kommunistischer Wahlerfolge abgesagt. Als sich auch 1933 ein politisch unerwünschtes Ergebnis abzeichnete ${ }^{92}$, wurden sie

${ }^{85}$ Vermerk über das Ergebnis des Vortrags am 8. 11. 1927 beim Herrn Minister, 12. 11. 1927, BAP RAM 501, Bl. 67.

86 Aktenvermerk, 10.12. 1927, BAP RAM 501, Bl. 182. Siehe auch Regierungserklärungen in der 229. Sitzung des 9. Ausschusses (Soziale Angelegenheiten) des Reichstages am 9.12. 1927, wo die Regierung erstmals massiv Bedenken gegenüber einer Hinzuziehung der Gewerkschaften äußert, BAP RAM 501, Bll. 206-208.

87 BAP RAM 501, Bl. 203.

88 Regierungserklärung 1.2. 1928, BAP RAM 501, Bl. 205.

89 Aktenvermerk 9. 2. 1928, BAP RAM 501, Bl. 195.

90 RGBI.I, 1928, S. 46. Kalckbrenner, Das Abänderungsgesetz zum Betriebsrätegesetz, S. 95 f.

91 Krause, Abänderung des Betriebsrätegesetzes, in: Der Arbeitgeber 18 (1928), S. 184. Ferner Anthes, Abänderung des Betriebsrätegesetzes, in: Deutsche Wirtschaftszeitung 25 (1928), S. 132. Siehe die Stellungnahme der VDA, zitiert bei Nörpel, 10 Jahre Betriebsrätegesetz in Deutschland, in: Gewerkschaftliche Rundschau für die Schweiz 22 (1930), S. 119.

92 Nachdem bereits in Sachsen und Thüringen die Reichskommissare die Betriebsrätewahlen ausgesetzt und kommunistischen Betriebsräten die Ausübung ihrer Funktion untersagt hatten, kabelte 
von den Landesbehörden kurzerhand abgebrochen, nachdem sie hierzu durch ein Gesetz ermächtigt worden waren, das nach einer nur gut vier Wochen dauernden Vorbereitungszeit am 4. April 1933 in Kraft trat: das Gesetz über Betriebsvertretungen und wirtschaftliche Vereinigungen. ${ }^{93}$ Dieses in der Literatur zur Frühgeschichte des Nationalsozialismus lange wenig beachtete Gesetz ${ }^{94}$ ist in seiner Wirkung für die Stabilisierung der NS-Herrschaft und die Einschüchterung der Arbeiterschaft kaum zu überschätzen, machte es doch letztlich die Beibehaltung des Arbeitsplatzes von der politischen Gesinnung abhängig. Ganz zu Recht wurde behördenintern mehrfach darauf verwiesen, daß dieses Gesetz in der Privatwirtschaft jene Funktion habe, die im öffentlichen Dienst das Gesetz über die Wiederherstellung des Berufsbeamtentums erfüllte: die politische Säuberung der Belegschaften und die Stellenfreimachung für Anhänger der NSDAP. Zugleich legalisierte es Repressionen gegen alle gewählten Betriebsräte, die keiner regierungstragenden Organisation angehörten, insbesondere ihre Entfernung aus dem Amt und ihre Entlassung.

Die Initiative zu diesem Gesetz ging nicht vom Reichsarbeitsministerium aus, das angesichts der seit 1932 laufenden Diskussionen um seine Auflösung bzw. Rückführung in das Reichswirtschaftsministerium ohnehin kaum noch handlungsfähig war. Sie folgte vielmehr der "Straße“. Unmittelbar nach der Machtergreifung begann im Februar 1933 ein Prozeß der Amtsverdrängung der mehrheitlich freigewerkschaftlichen Betriebsräte durch NSBO-Funktionäre und andere Nationalsozialisten. In der Regel zwang man die Betriebsräte zum Rücktritt und drängte danach die Unternehmensleitungen, die geschaßten Betriebsräte zu entlassen. Auch wenn manche Unternehmensleitungen diese Praktiken durchaus begrüßten, war ihnen zumeist der gesetzwidrige Charakter der Vorgänge durchaus bewußt. ${ }^{95} \mathrm{Da}$ die Arbeitsgerichte sich nicht ohne weiteres dem wilden Vorgehen der NSBO anschlossen, entstand eine prekäre rechtliche Situation, die das Reichsinnenministerium am 13.März 1933 veranlaßte, das Reichsarbeitsministerium aufzufordern, eine Notverordnung zur Änderung des Betriebsrätegesetzes vorzubereiten, die es ermöglichen sollte, die Bestimmung des $\$ 84$ des BRG zu umgehen, die einen Einspruch gegen eine Entlassung aus politischen Gründen für zulässig erklärte. ${ }^{96}$ Das Reichsarbeitsministerium hielt eine Änderung allein des $\$ 84$ BRG für wenig sinnvoll, da dieser ohnehin nicht angewendet würde und den entlassenen Arbeitern weiterhin ein Einspruch wegen „unbilliger Härte“ möglich

der Kölner Polizeipräsident am 17.3. 1933 seinen dringenden Wunsch nach Berlin, daß auch in Preußen die Wahlen bis auf weiteres verschoben werden müßten, da die Kommunisten versuchten, „in den Betrieben wieder Boden zu gewinnen ... Da ein Erfolg aus allgemeinpolitischen Gründen unerwünscht ist, möglicherweise Generalstreikgefahr droht, bitte zu erwägen (!), die Betriebsratswahlen auf längere Zeit zu verschieben..." Polizeipräsident Köln an. Preuß. Minister des Inneren, für Wirtschaft und Arbeit und an das Reichsarbeitsministerium, 17.3. 1933, BAP RAM 394, Bl. 10.

93 RGBI. I 1933, S. 161.

94 Erwähnung findet es bei Rüther, Arbeiterschaft in Köln, S. $114 \mathrm{ff}$. Vgl. auch Wisotzky, Der Ruhrbergbau im Dritten Reich, S. 33. Ausführlicher Frese, Betriebspolitik im „Dritten Reich“.

95 Vgl. beispielsweise die Vorgänge im Regierungsbezirk Düsseldorf, die durch die Gewerbeaufsicht en detail belegt sind, HStA Düsseldorf Reg. Düsseldorf 33642, 33643.

96 Reichsminister des Innern an den Herrn Reichsarbeitsminister, 13.3. 1933, BAP RAM 502, Bl. 121. 
wäre. Man war sich darüber im klaren, daß eine Säuberung der Betriebe insbesondere von Kommunisten nur erreicht werden könnte, „wenn ein ausdrückliches Recht zur Kündigung wegen kommunistischer Parteizugehörigkeit oder Betätigung geschaffen würde." 97

Das Reichsarbeitsministerium gedachte daher, der Reichsregierung per Notverordnung eine arbeitsrechtliche Generalermächtigung erteilen zu lassen. Am 18. März 1933 brachte man den Entwurf einer Notverordnung des Reichspräsidenten zur „Befriedung der Betriebe“ in das Kabinett ein, die lapidar besagte, daß die Reichsregierung ermächtigt werde, „aus Gründen der öffentlichen Sicherheit und Ordnung oder zur Erhaltung der Ruhe und des Friedens in den Betrieben Änderungen der Vorschriften des Betriebsrätegesetzes über die Wahlzeit, die Mitgliederzahl und die Auflösung der gesetzlichen Betriebsvertretungen, über das Erlöschen von Ämtern der gesetzlichen Betriebsvertretungen, über die Wahlen und die Wählbarkeit zu diesen Ämtern sowie über die Behördenzuständigkeit in solchen Angelegenheiten vorzunehmen. ${ }^{988}$ Parallel zur Vorbereitung der Ermächtigungsverordnung ${ }^{99}$ ging das Reichsarbeitsministerium daran, entsprechende Gesetzentwürfe auszuarbeiten. Einer eilig für den 20. März1933 einberufenen Ministerialbesprechung wurde zwei Tage später der Entwurf eines regelrechten Gewerkschaftsgesetzes vorgelegt, das in einem Zusatzartikel die Kündbarkeit aus politischen Gründen einführte. ${ }^{100}$ Der Gesetzentwurf hielt aber noch an der Weimarer Tradition fest, nur gegnerunabhängigen Vereinigungen die Tariffähigkeit zuzugestehen, wobei im Zweifelsfalle eine Spruchstelle beim Reichsarbeitsgericht über deren Tariffähigkeit zu entscheiden hatte. Zugleich ermächtigte er die Reichsregierung, die bisherigen Vertretungen der Arbeitnehmerschaft bei der Reichsknappschaft, der Reichsanstalt und den verschiedenen Versicherungsträgern auszutauschen sowie weiteren Organisationen die Vertretungsvollmacht vor den Arbeitsgerichten zu erteilen. Das Wirtschaftskabinett beschloß am 27. März 1933 indes einen anderen Entwurf. Offensichtlich wollte man sich in der Gewerkschaftsfrage nicht in einer Weise festlegen, die einer Bestandsgarantie gleichkam. Man war sich nach längerer Debatte einig, nichts zu tun, „was dem Ziel einer künftigen berufsständischen Neuordnung hinderlich sein oder vorgreifen könnte."101

Das Gesetz vom 4. April 1933 hob in seinen ersten beiden Artikeln die Wahlund Schutzvorschriften der Betriebsräte auf, ermöglichte die Amtsenthebung von Betriebsräten durch die Behörden, gab ihnen zugleich das Recht der Neueinsetzung von Betriebsvertretungen und bestimmte des weiteren, daß bei Entlassungen wegen „staatsfeindlicher Tätigkeiten“ ein Einspruchsrecht nicht beim Arbeitsge-

97 Antwortentwurf des Reichsarbeitsministeriums, März 1933, BAP RAM 502, Bl. 122.

98 Reichsarbeitsminister an den Staatssekretär der Reichskanzlei, 18.3. 1933, BAP RJM 2186, Bll. $107 \mathrm{f}$.

99 Diese arbeitsrechtliche Ermächtigungsverordnung wurde durch das Ermächtigungsgesetz überflüssig. Mit dem Ermächtigungsgesetz vom 23. 3. 1933 war die Reichsregierung in ihrer Gesetzgebung ohnehin weitgehend unabhängig vom Reichstag. Auf dieser Basis erfolgte auch der Beschluß über das Gesetz vom 4. 4. 1933. Vgl. Broszat, Der Staat Hitlers, S. $108 \mathrm{ff}$.

100 Reichsarbeitsminister an die Reichsministerien, 18. 3. 1933, BAP RJM 2186, Bll. 110-113.

101 Vermerk im Reichsjustizministerium über die Besprechung im wirtschaftspolitischen Ausschuß des Kabinetts vom 27. 3. 1933, BAP RJM 2186, Bl. 114. 
richt, sondern lediglich gegenüber der zuständigen Landesbehörde bestand, die nach Anhörung der Beteiligten über die Entlassung zu entscheiden hatte. Zugleich änderte das Gesetz die Vertretungsrechte in der Reichsknappschaft und vor Arbeitsgerichten. ${ }^{102}$ Das Betriebsrätegesetz war damit in seiner materiellen Substanz nicht betroffen. Rechte und Pflichten der Betriebsvertretungen blieben formell unangetastet; lediglich die Zusammensetzung der Betriebsvertretungen wurde dem behördlichen Zugriff in einer Weise geöffnet, daß der eingetretene Zustand "legalisiert" werden konnte. Hierüber war man sich bei einer Besprechung im Reichsarbeitsministerium am 11. April $1933^{103}$, die sich mit der praktischen Durchführung des Gesetzes befaßte, weitgehend im klaren: „Soweit vor Inkrafttreten des Gesetzes Mitglieder von Betriebsvertretungen durch nichtbehördliche Stellen abgesetzt und durch neue Mitglieder ersetzt sind, wird es Aufgabe der nunmehr zuständigen Behörde [in Preußen der Gewerbeaufsicht bzw. des Regierungspräsidenten] sein, für eine dem Gesetz entsprechende Betriebsvertretung zu sorgen. Dabei wird es nach der vorherrschenden Meinung der Ressortvertreter das Ziel sein müssen, die bestehenden tatsächlichen Zustände möglichst weitgehend zu legalisieren, zumal es sich nur um eine Übergangsregelung handele, auf Erhaltung des Wirtschaftsfriedens besonderes Gewicht zu legen sei und dem Arbeitnehmer sein Arbeitsplatz im Betrieb erhalten bleibe", sofern er nicht unter eine der zuvor definierten Kategorien von Staatsfeinden fiel. Hierzu zählten Mitglieder oder Aktivisten der kommunistischen Bewegung automatisch, Mitglieder der SPD und der freien Gewerkschaften bei Vorliegen besonderer Umstände (i.e. etwa Befürwortung der Einheitsfront). Bei der Entscheidung sollten auch Geschehnisse aus der Vergangenheit herangezogen werden. Bei Personen „nichtarischer Abstammung" genügte dieser Umstand als Entlassungsgrund allein nicht, so zumindest die Auffassung der versammelten Beamten. Wenn eine Mitgliedschaft in der SPD oder bei den freien Gewerkschaften eine Entlassung auch nicht automatisch rechtfertige, so „könne an eine weitere Auslegung des Begriffes der Wirtschaftsfeindlichkeit zur Beseitigung von Betriebsvertretungsmitgliedern...gedacht werden", wobei sinnvollerweise nach der Art des Betriebes (Rüstung etc.) zu differenzieren sei.

Die Landesbehörden entließen zügig Ausführungsbestimmungen ${ }^{104}$, mit denen sie die Zuständigkeiten bei Widersprüchen regelten und zugleich die Betriebsratswahlen für das nächste halbe Jahr "aussetzten“. ${ }^{105}$ Damit war klar, daß freigewerkschaftliche Betriebsratsmitglieder ihrer Ämter verlustig gehen würden, sofern dies nicht bereits geschehen war. Zugleich gerieten alle ehemaligen $\mathrm{Be}-$ triebsvertretungsmitglieder und kommunistischen, sozialdemokratischen ${ }^{106}$ und

${ }^{102}$ RGBL. I 1933, S. $161 \mathrm{f}$.

${ }^{103}$ Niederschrift über die Besprechung mit den Vertretern der Reichsressorts und der Länder am 11. 4. 1933 über die Durchführung des Gesetzes über Betriebsvertretungen und über wirtschaftliche Vereinigungen, BAP RJM 2186, Bll. 129-132.

${ }^{104}$ Preußische Gesetzsammlung 15. 4. 1933, Nr. 27, S. 109.

105 Ähnlich verfuhren die Reichsbehörden, die ebenfalls für ihren Zuständigkeitsbereich die Wahlen "aussetzten“. Runderlaß des Reichsjustizministers, 9. 5. 1933, BAP RJM 2186, Bl. 133.

${ }^{106}$ In einem Erlaß für Preußen erklärte Göring im Juni 1933 nach dem faktischen Verbot der SPD SPD-Mitglieder für Staatsfeinde im Sinne des Gesetzes vom 4.4. 1933, Broszat, Staat Hitlers, S. 120 . 
„nichtarischen“ Belegschaftsangehörigen unter akuten Entlassungsdruck, wobei gegen Entlassungen nur noch bei der Gewerbeaufsicht Einspruch eingelegt werden konnte, den diese allerdings nach Gutdünken entscheiden konnte. Unter dem akuten Druck der verschiedenen Nazi-Organisationen fielen die Entscheidungen in der Regel gegen die Einsprucheinlegenden, zumal sich in den Betrieben ein weitreichendes Denunziationsklima breitmachte. ${ }^{107}$ Noch im Dezember 1933 schrieb der Treuhänder der Arbeit für Sachsen: „Im Bereich der Kreishauptmannschaft [i.e. Regierungsbezirk] Leipzig wird Artikel 2 [Entlassung wg. staatsfeindlicher Betätigung]des Gesetzes noch immer mit unverminderter Schärfe zur Anwendung gebracht. Die Kreishauptmannschaft beklagt sich darüber, daß nach den Weisungen des Ministeriums die Zugehörigkeit zur SPD oder zum Reichsbanner allein nicht ausreichen soll, die Annahme staatsfeindlicher Tätigkeit zu begründen. Der Kreishauptmann in Leipzig (lehnt) im wesentlichen alle Einsprüche ab. " 108

Die Bedeutung des Gesetzes war eine doppelte. Zum einen legalisierte es innerhalb weniger Wochen den völligen Austausch der Betriebsräte. An die Stelle der gewählten Betriebsvertretungen traten NSBO-Leute und/oder andere Parteigänger des Nationalsozialismus, die im übrigen auch vor der Verdrängung gewählter Stahlhelm-Vertreter oder Werkvereinler nicht zurückschreckten. ${ }^{109}$ Zugleich wurden Kommunisten und Sozialdemokraten in großer Zahl aus den Betrieben entlassen oder gerieten unter Entlassungsdruck, der jede politische Äußerung zu einer existentiellen Frage machen konnte. Gerade der Entlassungsdruck wirkte ausgesprochen disziplinierend, wie der Verband der mitteldeutschen Industrie mit dem Sitz in Weimar im November 1933 feststellte: „Der innere Widerstand der Belegschaft [bei Zeiss und Schott] gegen die zu fördernde nationalsozialistische Wirtschafts- und Volksgemeinschaft erklärt sich u. a. daraus, daß der überwiegende Teil der Belegschaft ... auf Grund des für Zeiß und Schott gültigen Stiftungsstatuts nach 5jähriger Betriebszugehörigkeit pensionsberechtigt wird und nur aus wichtigem Grunde ... außerordentlich kündbar ist." Die mitteldeutsche Industrie bedauerte daher, daß das Gesetz vom April 1933 in die Satzungsvorschriften nicht eingriff und regte eine entsprechende Korrektur an. ${ }^{110} \mathrm{Zum}$ anderen brachte das Gesetz die „spontane Landnahme“ der Nationalsozialisten in den Betrieben in eine legale Form und kanalisierte sie zugleich, indem sie Absetzungen, Neueinsetzungen und Entlassungen einem förmlichen Verfahren unterzog, durch das erst die Rechtsgültigkeit der entsprechenden Maßnahmen hergestellt wurde. Es beseitigte dabei nicht die Rechte der Betriebsvertretungen, sondern stellte eben nur sicher, daß sie von ausgemachten Nationalsozialisten wahrgenommen wurden.

${ }^{107}$ Siehe einzclne Fälle in: HStA Düsseldorf Reg. Düsseldorf 33642, 33643; Reg. Köln 8287, 8288, 8289.

${ }^{108}$ Treuhänder der Arbeit für das Wirtschaftsgebiet Sachsen an Reichsarbeitsministerium 30. 12. 1933, BAP RAM 505, Bll. 344-346.

${ }^{109}$ Schreiben des Kreisverbandes Dortmund der DNVP an das Reichsarbeitsministerium, 10.4. 1933, BAP RAM 505, Bl. 110.

110 Verband der mitteldeutschen Industrie an Reichsarbeitsministerium, 7.11. 1933, BAP RAM 505, Bll. 303-304. 
Die Beseitigung der Betriebsvertretungsrechte erfolgte erst mit dem Gesetz zur Ordnung der nationalen Arbeit vom Januar 1934, dessen Entstehungsgeschichte aufgrund umfangreicher Aktenverluste bei Kriegsende im Dunkeln liegt. ${ }^{111}$ An die Stelle der Betriebsräte traten vom Betriebsführer und der Deutschen Arbeitsfront (DAF) abhängige Vertrauensräte ${ }^{112}$, die Aufsichtsratsvertretung wurde durch diffuse Unternehmensbeiräte ersetzt, die Möglichkeit zur gerichtlichen Konfliktregulierung zugunsten einer Ehrengerichtsbarkeit aufgehoben, die mit der alten Arbeitsrechtsprechung kaum etwas zu tun hatte. Die Arbeitsgerichtsbarkeit blieb im übrigen erhalten. ${ }^{113}$ Der Betrieb wurde - in gewisser Weise einer spezifischen Interpretation der schon in Weimar verbreiteten Werks- oder Betriebsgemeinschaftsvorstellung entsprechend ${ }^{114}$ - als Einheit von Führer und Gefolgschaft definiert, die gemeinsam das Wohl des Betriebes zu fördern und damit zum Volkswohl beizutragen hatten. ${ }^{115}$ Das nationalsozialistische Arbeitsrecht, dessen weiterer Eckstein die Treuhänder der Arbeit waren, die im Mai 1933 an die Stelle des alten Tarifsystems traten ${ }^{116}$, war mithin nicht von einer Rückkehr zum arbeitsrechtlichen "Liberalismus" des Kaiserreichs gekennzeichnet, sondern baute Elemente des kollektiven Arbeitsrechtes (Tarife, kollektive Vertretungen etc.) in ein System konfliktfrei gedachter Gemeinschaftsordnung ein, etablierte also eine Art klassenkampffreie und rassistische Gemeinschaftsordnung mit Sozialbindung aller Beteiligten. Referenzebene von Kommunikation und Handeln war nunmehr anders als im Betriebsrätegesetz ausschließlich das Betriebswohl, das seinerseits aber als Teil des Volkswohls gesehen und damit keineswegs nur unter partikulärem Unternehmergesichtspunkt verstanden wurde. Mit der Negierung von Partikularinteressen bzw. ihrer Ausgrenzung als wie auch immer schädlich war zwangsläufig keine Notwendigkeit für entsprechende Interessenvertretungsorgane, also auch nicht für entsprechende Kommunikation vorhanden. Insofern war die Beseitigung autonomer Interessenvertretungen im AOG folgerichtig. Die auf diese Weise generierten defekten Kommunikationsstrukturen mochten funktionieren, solange der Arbeitsmarkt der spontanen Interessenvertretung der abhängig Beschäftigten Grenzen setzte. Sobald sich hier allerdings Änderungen ergaben, war zu erwarten, daß die Unterdrückung kollektiver Konfliktregulierung zu verstärkten individuellen Konfliktstrategien führen würde. ${ }^{117}$

Freilich ist aus dem Text des AOG keineswegs darauf zu schließen, wie sich die industriellen Beziehungen im Betrieb nach 1934 entwickelten. Schon die weitgehende Verdrängung freigewerkschaftlicher Betriebsräte nach dem März 1933

111 Mason, Zur Entstehung des Gesetzes zur Ordnung der nationalen Arbeit, S. 329 f.

112 Öhm, Vom Betriebsrat zum Vertrauensrat, in: Soziale Praxis 43 (1934), Sp. 519-524.

${ }^{113}$ Bähr, Arbeitsgerichtsbarkeit, S. $527 \mathrm{ff}$.

114 Sowohl Mason, Zur Entstehung des Gesetzes zur Ordnung der nationalen Arbeit, passim, als auch Bähr, Arbeitsgerichtsbarkeit, S. $529 \mathrm{ff}$ weisen auf die Nähe des AOG zu Weimarer Traditionen hin.

115 Rüthers, Dic Betriebsverfassung im Nationalsozialismus, in: Arbeit und Recht 18 (1970), S. $97-$ 109. Vgl. auch Spohn, Zur „Betriebsverfassung“, in: Gewerkschaftliche Monatshefte 35 (1984), S. 545-555.

${ }^{116}$ Zur Vorgeschichte des Gesetzes, insbesondere zur Diskussion über „Reichsbeauftragte zur Befriedung des Arbeitslebens“ finden sich einige Aktenreste im BAP, RJM 2178, Bll. $382 \mathrm{ff}$. Zum Gesetz selbcr siehe Hachtmann, Industriearbeit im „Dritten Reich“, S. $32 \mathrm{ff}$.

117 Hachtmann, Die Krise der nationalsozialistischen Arbeitsverfassung, in: Kritische Justiz 17 (1984), S. 281-299. Allgemein Kranig, Lockung und Zwang. 
mußte keineswegs ein Ende autonomer Betriebsratstätigkeit zur Folge haben, sondern konnte ohne weiteres mit einem Festhalten an der bisherigen Interessenvertretungspolitik - freilich jetzt mit anderer Begründung - verbunden sein. Zweitens wuchs mit dem AOG und wohl auch bereits zuvor im Rahmen der Weltwirtschaftskrise die Macht der Betriebsleitungen zur autonomen Regelung der Arbeitsorganisation. Ob und wie weit diese neuen Möglichkeiten genutzt wurden oder eher an erprobten Kooperationsformen festgehalten wurde, wird weiter unten zu diskutieren sein. Als man etwa in der nordwestdeutschen Eisenund Stahlindustrie im Gefolge des AOG an eine Neufassung der Arbeitsordnungen und ihre Überführung in gesetzeskonforme Betriebsordnungen ging, war man sich in der einschlägigen Kommission von Arbeitnordwest schnell einig: „Es wird noch besonders darauf hingewiesen, daß man bei der Aufstellung des Entwurfs der Betriebsordnung von dem Gedanken ausgegangen sei, die alte [noch mit den Betriebsräten vereinbarte] Arbeitsordnung ohne große Änderungen zu übernehmen, da diese sich in der jahrelangen Praxis bewährt habe. " ${ }^{118}$ Dieser Entwurf einer Arbeitsordnung unterschied sich fundamental von einem rassistisch-nazistischen Machwerk, das zu gleicher Zeit das DINTA als Vorlage für die Neufassung der Arbeitsordnungen vorgelegt hatte. ${ }^{19}$ Ganz ähnlich war die Entwicklung im Ruhrbergbau, wo sich die Unternehmen mit ihrem an die Arbeitsordnung von 1921 angelehnten Entwurf einer neuen Betriebsordnung gegen die Wünsche der DAF nach einer expliziten Nazifizierung der industriellen Beziehungen im Betrieb durchsetzen konnten. ${ }^{120}$ Für die Unternehmen - so eine mögliche These ging es 1933 um die (Wieder-)Herstellung ihrer Handlungsautonomie gegenüber den Belegschaften, was aber keineswegs mit einer ideologischen Nazifizierung des Arbeitsverhältnisses oder der Arbeitsbeziehungen verwechselt werden sollte. ${ }^{121}$ Insofern determinierte auch das AOG nicht die betriebliche Praxis der Arbeitsbeziehungen, sondern führte vor allem dazu, daß die grundlegende betriebliche Machtasymmetrie zugleich in jedem einzelnen Kommunikations- und Handlungsakt im Betrieb mobilisierbar wurde. Wie die Unternehmen ihre Macht konkret nutzten, war freilich eine andere Frage.

\section{Zusammenfassung}

Resümiert man die Entwicklung der gesetzlichen Vorschriften, so lassen sich zwei Punkte festhalten.

1. In einem vor allem von politischen Krisen und Umbrüchen gekennzeichneten Gesetzgebungsprozeß wurde zwischen 1916 und 1920 eine neue Betriebsverfassung geschaffen, die auf der Basis der bestehenden Wirtschaftsordnung die Lei-

\footnotetext{
${ }^{118}$ Niederschrift über die Sitzung der Betriebsordnungskommission von Arbeitnordwest vom 8.6. 1934, ThA VSt $/ 460$.

119 Mitteilungsblatt DINTA 9/1934: Entwurf vom 15.5. 1934. Entwurf von Arbeitnordwest vom 22. 5. 1934, siehe die Unterlagen in: ThA VSt/461.

120 Wisotzky, Der Ruhrbergbau im Dritten Reich, S. 45-50.

121 Vgl. die Uberlegungen von Hachtmann, Von der Klassenharmonie zum regulierten Klassenkampf, S. $159-183$.
} 
stungskrise der deutschen (Groß-)Industrie durch verstärkte Partizipation der Arbeitnehmer an den betrieblichen Entscheidungsprozessen lösen wollte. Im Kern griff die 1920 endgültig kodifizierte Betriebsverfassung daher das Konzept der „bürgerlichen“ Sozialreform und der christlichen Gewerkschaftsbewegung aus der Vorkriegszeit auf. Nachdem sich dieses Konzept in der Gesetzgebung durchgesetzt hatte, blieb es de facto bis 1933/34 unverändert bindend. Die nationalsozialistische Arbeitsverfassung brachte einen radikalen Bruch, insofern sie den Ansatz der Partizipation der Arbeitnehmerschaft beseitigte und die notwendige Akzeptanz von Entscheidungen im konfliktkonstituierten Handlungsfeld Betrieb durch ideologische Appelle an die Beschäftigten ersetzte, die der ständigen Repressionsdrohung bedurften, um wirksam zu sein. Eine Kontinuität der "Gemeinschaftskonzepte" von der Weimarer Zeit in den Nationalsozialismus hinein bestand zumindest von der gesetzlichen Seite her nur scheinbar.

2. Die politischen und sozialen Rahmenbedingungen des Gesetzgebungsprozesses zwischen 1916 und 1920 führten zu einer starken ideologischen Aufladung der zukünftigen Betriebsverfassung und bedingten eine Kontroverse im Gesetzgebungsprozeß, die allerdings nach 1920 rasch beendet war. Ernsthafte Ansätze, zu einer grundlegenden Reform der Betriebsverfassung zu kommen bzw. das Betriebsrätegesetz wieder zu beseitigen, gab es vor 1933 nicht. Nicht nur die Befürworter des Betriebsrätegesetzes verzichteten aus letztlich pragmatischen Gründen auf das Einbringen von Reformvorschlägen. Auch seine schärfsten Kritiker von Seiten der Arbeitgeber und der linksradikalen Teile der Arbeiterbewegung wurden zunehmend zurückhaltender, selbst wenn sich seit 1928 erneut Ansätze zeigten, die bestehende Betriebsverfassung in Frage zu stellen. Gesetzgeberisch wirksam wurden sie zu keinem Zeitpunkt. 